\title{
The long-lived Type IIn SN 2015da: Infrared echoes and strong interaction within an extended massive shell ${ }^{\star, \star \star}$
}

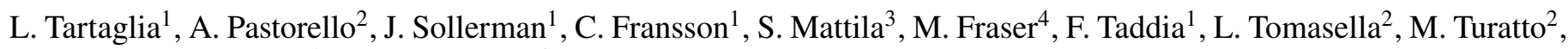 \\ A. Morales-Garoffolo ${ }^{5}$, N. Elias-Rosa ${ }^{2,6,7}$, P. Lundqvist ${ }^{1}$, J. Harmanen ${ }^{3}$, T. Reynolds ${ }^{3}$, E. Cappellaro ${ }^{2}$, C. Barbarino ${ }^{1}$, \\ A. Nyholm ${ }^{1}$, E. Kool ${ }^{1}$, E. Ofek ${ }^{8}$, X. Gao ${ }^{9}$, Z. Jin ${ }^{10}$, H. Tan ${ }^{11}$, D. J. Sand ${ }^{12}$, F. Ciabattari ${ }^{13}$, X. Wang ${ }^{14}$, \\ J. Zhang ${ }^{15,16}$, F. Huang ${ }^{17,14}$, W. Li $^{14}$, J. Mo ${ }^{14}$, L. Rui ${ }^{14}$, D. Xiang ${ }^{14}$, T. Zhang ${ }^{18,19}$, G. Hosseinzadeh ${ }^{20}$, D. A. Howell ${ }^{21,22}$, \\ C. McCully ${ }^{21}$, S. Valenti ${ }^{23}$, S. Benetti ${ }^{2}$, E. Callis ${ }^{4}$, A. S. Carracedo ${ }^{24}$, C. Fremling ${ }^{25}$, T. Kangas ${ }^{26}$, A. Rubin ${ }^{27}$, \\ A. Somero $^{3}$, and G. Terreran ${ }^{28}$
}

(Affiliations can be found after the references)

Received 22 August 2019 / Accepted 29 December 2019

\begin{abstract}
In this paper we report the results of the first $\sim$ four years of spectroscopic and photometric monitoring of the Type IIn supernova SN 2015da (also known as PSN J13522411+3941286, or iPTF16tu). The supernova exploded in the nearby spiral galaxy NGC 5337 in a relatively highly extinguished environment. The transient showed prominent narrow Balmer lines in emission at all times and a slow rise to maximum in all bands. In addition, early observations performed by amateur astronomers give a very well-constrained explosion epoch. The observables are consistent with continuous interaction between the supernova ejecta and a dense and extended $\mathrm{H}-$ rich circumstellar medium. The presence of such an extended and dense medium is difficult to reconcile with standard stellar evolution models, since the metallicity at the position of SN 2015da seems to be slightly subsolar. Interaction is likely the mechanism powering the light curve, as confirmed by the analysis of the pseudo bolometric light curve, which gives a total radiated energy $\gtrsim 10^{51} \mathrm{erg}$. Modeling the light curve in the context of a supernova shock breakout through a dense circumstellar medium allowed us to infer the mass of the prexisting gas to be $\simeq 8 M_{\odot}$, with an extreme mass-loss rate for the progenitor star $\simeq 0.6 M_{\odot} \mathrm{yr}^{-1}$, suggesting that most of the circumstellar gas was produced during multiple eruptive events. Near- and mid-infrared observations reveal a flux excess in these domains, similar to those observed in SN 2010jl and other interacting transients, likely due to preexisting radiatively heated dust surrounding the supernova. By modeling the infrared excess, we infer a mass $\gtrsim 0.4 \times 10^{-3} M_{\odot}$ for the dust.
\end{abstract}

Key words. supernovae: general - galaxies: individual: NGC 5337 - supernovae: individual: PSN J13522411+3941286 supernovae: individual: iPTF16tu - supernovae: individual: SN 2015da

\section{Introduction}

Supernovae ( $\mathrm{SNe}$ ) interacting with a preexisting dense circumstellar medium (CSM) belong to an intriguing and not fully understood class of transients, including the Ibn (Pastorello et al. 2008) and IIn (Schlegel 1990) classes. Early optical spectra of Type IIn $\mathrm{SNe}$ show a blue continuum with narrow (from a few tens to $\simeq 10^{3} \mathrm{~km} \mathrm{~s}^{-1}$ ) Balmer lines in emission, and broad wings resulting from electron scattering in an ionized, unshocked, $\mathrm{H}-$ rich CSM (see, e.g., Chugai 2001). These lines, visible at all phases of the spectroscopic evolution, are the signature of underlying interaction between SN ejecta and slow moving CSM, as they are the result of recombination in the outer unshocked layers, ionized by photons emitted in the shocked regions. When the fast-moving ejecta hit the preexisting CSM, forward/reverse shocks form at the interface between the two media (also producing a contact discontinuity and a "cool dense shell" - CDS;

\footnotetext{
* Tables A.1-A.4 are only available at the CDS via anonymous ftp to cdsarc.u-strasbg.fr $(130.79 .128 .5)$ or via http://cdsarc. u-strasbg.fr/viz-bin/cat/J/A+A/635/A39.

${ }^{\star \star}$ Spectroscopic data and photometric tables are available through the Weizmann Interactive Supernova Data Repository (WISeREP) at the following address: https://wiserep.weizmann.ac.il/object/ 13868 .
}

Fransson 1984; Chevalier \& Fransson 1994), and high energy photons propagate in both directions, either ionizing the freely expanding SN ejecta or the slow-moving CSM.

Under specific conditions (e.g., particular geometrical configurations), the resulting emission lines can be characterized by structured, asymmetric, and multicomponent profiles, produced by recombining gas shells moving at different velocities (see, e.g., the case of the prototypical SNe 1987F; Wegner \& Swanson 1996 and 1988Z; Stathakis \& Sadler 1991; Turatto et al. 1993), although in other cases, the overall profiles are characterized by pure Lorentzian profiles at all epochs (see, e.g., the case of SN 2010j1; Fransson et al. 2014). Depending on the density of the CSM, the early light curve of IIn SNe might be dominated by photon diffusion rather than ${ }^{56} \mathrm{Ni}$ decay (e.g., Balberg \& Loeb 2011), possibly extending the "shock breakout" signal (e.g., Ofek et al. 2010; Chevalier \& Irwin 2011). Modeling the bolometric light curves of SNe exploding in a dense wind allows us to infer crucial information about the nature of the exploding star and its environment (such as the mass-loss rate and the mass of the surrounding CSM; e.g., Balberg \& Loeb 2011; Svirski et al. 2012). Narrow lines, if resolved, may also be used to infer wind speeds directly from their P Cygni absorption features.

Type IIn SNe are relatively rare ( $~ 9 \%$ of all Type II, H-rich core-collapse SNe-CC SNe; Li et al. 2011), although they are 
more common than Ibn SNe. They show a remarkable heterogeneity, with absolute peak magnitudes ranging from $M_{\mathrm{r}}=-17$ to -22 mag (Kiewe et al. 2012; Taddia et al. 2013b), with a mean rise time of $\simeq 17 \mathrm{~d}$ (based on the sample of 15 objects published in Ofek et al. 2014a). On the other hand, this value might be strongly affected by the limited number of SNe IIn discovered soon after explosion and/or well-studied transients, whose publication is likely biased towards the most peculiar or luminous objects (see, e.g., SN 2006gy; Ofek et al. 2007; Smith et al. 2007, and the "super-luminous" Type II SNe - SLSN II; Gal-Yam 2019). A recent analysis on a greater sample of $42 \mathrm{SNe}$ IIn discovered by the Palomar Transient Factory (PTF) reveals an average peak luminosity of $M_{\mathrm{r}}=-19.18 \pm 1.32 \mathrm{mag}$ and a bimodal distribution of rise times, with $t_{\text {rise }}=19 \pm 8 \mathrm{~d}$ and $50 \pm 15 \mathrm{~d}$ (Nyholm et al. 2020). A wide range of photometric properties is also observed after peak, with a fraction of IIn SNe (e.g., SNe 1988Z; Stathakis \& Sadler 1991; Turatto et al. 1993, 2005ip and 2006jd; Stritzinger et al. 2012) showing a very slow evolution, with the transient still visible several years after explosion (see, e.g., SN 1995N; Fransson et al. 2002; Pastorello et al. 2005). In other cases, light curves exhibit a plateau-like shape with steep post-plateau declines (IIn-P; Kankare et al. 2012; Mauerhan et al. 2013) or otherwise almost "linear" declines (see, e.g., the case of SN 1999el; Di Carlo et al. 2002). Long-lasting Type IIn SNe are generally brighter than IIn-P, although not as bright as SLSNe.

The controversy surrounding the nature of the progenitors of SNe IIn is still not completely solved. The presence of an extended and dense CSM at the time of the explosion requires strong long-term winds, binary interactions, or giant eruptive events similar to those occasionally experienced by luminous blue variables (LBVs; Vink 2012, and references therein). Some observational evidence points to LBVs as progenitors of at least some Type IIn SNe. The direct observation of the likely quiescent progenitors of SNe 2005gl (Gal-Yam \& Leonard 2009) and 2009ip (Smith et al. 2010) in deep archival Hubble Space Telescope (HST) images strongly supports this scenario, as well as the disappearance of the LBV candidate progenitor of SN $2005 \mathrm{gl}$ in a post-explosion HST image obtained when the SN had faded (Gal-Yam \& Leonard 2009). Nevertheless, pre$\mathrm{SN}$ super-winds might not be rare in red supergiant (RSG) stars (see, e.g., the case of the Galactic RSG VY Canis Majoris; Smith et al. 2009a) suggesting that they are viable progenitors for at least a fraction of SNe IIn (as proposed for SN 1995N; Fransson et al. 2002; Pozzo et al. 2004). In addition, Tartaglia et al. (2016) showed that also stars less massive than LBVs can experience major eruptive events. Some observational evidences seem to suggest thermonuclear explosions of stars embedded in a dense and extended CSM as viable mechanisms to produce some Type IIn SNe. These Ia-CSM SNe (see, e.g., Silverman et al. 2013; Fox et al. 2015a) show optical spectra dominated by prominent narrow Balmer lines in emission and luminosities usually exceeding those typically observed in normal $\mathrm{SNe}$ Ia. A number of transients have been proposed to belong to this class (see, e.g., Silverman et al. 2013), although their real nature is still a matter of discussion (see, e.g., Benetti et al. 2006; Trundle et al. 2008; Inserra et al. 2016). On the other hand, the analysis performed on high-resolution optical spectra of PTF11kx (Dilday et al. 2012) revealed features first resembling those of the overluminous Type Ia SN 1999aa (see, e.g., Garavini et al. 2004, and references therein), but soon developing strong narrow $\mathrm{H} \alpha$ features, therefore suggesting that a fraction of SNe showing narrow emission features arise from thermonuclear explosions.

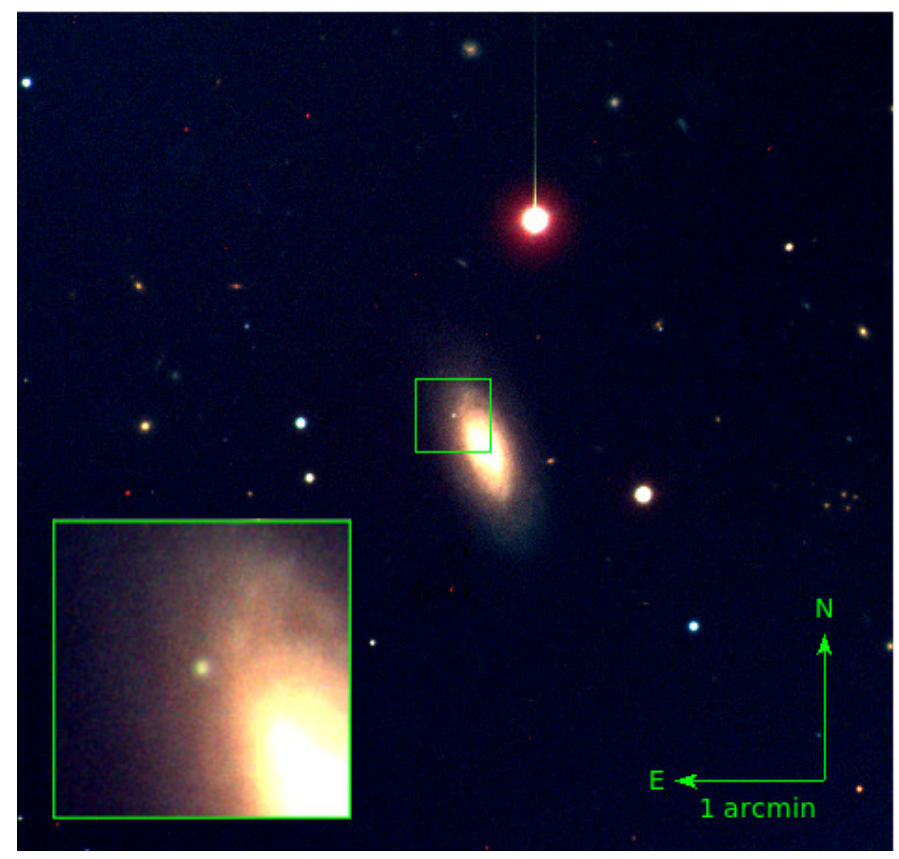

Fig. 1. Combined gri images of the field of NGC 5337 obtained on 2019 January 23 with the Nordic Optical Telescope with ALFOSC. The position of SN 2015da, still clearly visible, is highlighted within a green box. The insert shows SN 2015da, which is the bright source within the zoomed-in region.

More than $50 \%$ of SNe showing late (or very late, see, e.g., SNe 1999el; Di Carlo et al. 2002 and 2005ip; Fox et al. 2009, 2010, among others) infrared (IR) excess belong to the IIn class (see Gerardy et al. 2002; Fox et al. 2011), although this percentage might be biased by the still limited number of well studied $\mathrm{SNe}$ IIn in the IR domain. This suggests the presence of either preexisting or newly formed dust. Dust can, in fact, form in the rapidly cooling post-shock layers (e.g., Pozzo et al. 2004; Mattila et al. 2008) or be located in the dense preexisting CSM, becoming visible after being shock- or radiatively heated, or because echoing and reprocessing some of the SN light into IR radiation (e.g., Fox et al. 2010; Stritzinger et al. 2012; Fransson et al. 2014). Modeling the IR emission of SNe IIn showing late-time excesses can independently infer crucial information on the environments of progenitor stars as well as their mass-loss rates (e.g., Fox et al. 2010).

In this context, we report the results of our study of the longlasting Type IIn SN 2015da. The SN was discovered on 2015 January 9.90 UT in the nearby spiral galaxy NGC 5337 by Z. Jin and $\mathrm{X}$. Gao ${ }^{1}$, with an apparent magnitude $\simeq 18 \mathrm{mag}$, and was later classified as a Type IIn SN (Zhang \& Wang 2015). No source was detected at the position of the transient in an unfiltered image obtained on 2015 January 7 to a limiting magnitude $19.5 \mathrm{mag}$, or in previous frames of the field. SN 2015da is located at $\mathrm{RA}=13: 52: 24.11, \mathrm{Dec}=+39: 41: 28.2$ [J2000], 12'.54 E, 14"'04 N from the center of NGC 5337 (assuming RA=13:52:23.024, Dec $=+39: 41: 14.16-\mathrm{J} 2000-$ for the center of the host; Skrutskie et al. 2006, see also Fig. 1). The SN was extensively imaged by amateur astronomers, who provided very good sampling of the rise to the maximum through unfiltered images, later calibrated to the $R$-band. SN 2015 da was also followed by a

http://www . cbat .eps . harvard. edu/unconf/followups/ J13522411+3941286.html 
number of facilities and collaborations, such as the Nordic Optical Telescope Un-biased Transient Survey $\left(\right.$ NUTS $\left.^{2}\right)$ and its extension NUTS2, and the intermediate Palomar Transient Facility $^{3}$ (iPTF) under the designation of iPTF16tu. The discovery of SN 2015da was also reported by Petropoulou et al. (2017), who estimated a distance of $32.1 \mathrm{Mpc}$ based on the redshift derived from the heliocentric recessional velocity of NGC 5337 $\left(2165 \pm 17 \mathrm{~km} \mathrm{~s}^{-1}\right.$; van Driel et al. 2001), and a cosmology with $\Omega_{\mathrm{M}}=0.31, \Omega_{\Lambda}=0.69$, and $H_{0}=69.9 \mathrm{~km} \mathrm{~s}^{-1} \mathrm{Mpc}^{-1}$.

The paper is organized as follows: Sect. 2 describes the host galaxy, metallicity, and star formation rate at the location of SN 2015da, including a discussion on the local reddening. In Sect. 3, we report our analysis on data collected during the photometric (Sect. 3.1) and spectroscopic (Sect. 3.2) follow-up campaigns, and give a qualitative interpretation of the observed quantities. A summary of the main results of the paper is reported in Sect. 4, while in the appendix, we detail the observations and data-reduction techniques, provide the magnitude tables, and main information on the spectra.

\section{The host galaxy and local extinction}

NGC 5337 is a SBab galaxy, with a total corrected ${ }^{4}$ apparent $B$-band magnitude of $12.94 \pm 0.29 \mathrm{mag}$ and mean heliocentric radial velocity $(c z) V_{\mathrm{r}}=2127 \pm 2 \mathrm{~km} \mathrm{~s}^{-1}$ (as reported in the HyperLeda database ${ }^{5}$ ). In the following, we assume a luminosity distance of $53.2 \pm 13.2 \mathrm{Mpc}$ (corresponding to a distance modulus of $33.63 \pm 0.54 \mathrm{mag}$ ), which is the most recent redshift-independent distance estimate for NGC 5337 (Tully et al. 2016). We note, however, a large discrepancy among different distances reported in the literature for NGC 5337 (in particular among redshift-dependent distances reported in the NASA/IPAC Extragalactic Database $-\mathrm{NED}^{6}$ ). Assuming a distance modulus $\mu=33.63 \pm 0.54 \mathrm{mag}$ implies a total absolute magnitude of $B=-20.69 \pm 0.61 \mathrm{mag}$. The main properties inferred for SN 2015da and its environment described in this section are reported in Table 1.

An estimate of the local metallicity in the environment of SN 2015da can be inferred through the analysis of the spectral emission lines of a relatively nearby $\mathrm{HII}$ region, SDSS J135223.63+394136.2, located at RA=13:52:23.63, $\mathrm{Dec}=+39: 41: 36.21$ [J2000] (i.e., 5'.54 W, 8'. $01 \mathrm{~N}$ from the position of SN 2015da, corresponding to a projected distance of $2.51 \mathrm{kpc}$ at $53.2 \mathrm{Mpc}$ ). The spectrum of the $\mathrm{H}$ II region, available through the Sloan Digital Sky Survey Data Release 14 (SDSS DR14; Adelman-McCarthy et al. 2006) was obtained through the SDSS Catalog Archive Server $\left(\mathrm{CAS}^{7}\right)$. After correcting the spectrum for the foreground Galactic extinction, we estimated the internal reddening through the observed Balmer decrement, assuming an intrinsic ratio of 2.86 (case B recombination scenario, see Osterbrock \& Ferland 2006) and a standard extinction law with $R_{V}=3.1$ (Cardelli et al. 1989). Following Botticella et al. (2012), we therefore corrected the spectrum of the H II region by including an additional contribution of $E(B-V)=$ $0.47 \mathrm{mag}$ to the total color excess.

\footnotetext{
2 http://csp2.lco.cl/not/

3 https://www.ptf.caltech.edu/iptf

4 Apparent magnitude corrected for foreground Galactic extinction in the direction of NGC $5337\left(A_{\mathrm{B}, \mathrm{MW}}=0.052 \mathrm{mag}\right.$; Schlafly \& Finkbeiner 2011), internal extinction $\left(A_{\mathrm{B}, \mathrm{int}}=0.35 \mathrm{mag}\right.$; Bottinelli et al. 1995) and $\mathrm{k}$-correction.

5 http://leda.univ-lyon $1 . f r /$

6 https://ned.ipac.caltech.edu/

7 https://www.sdss.org/dr14/data_access/tools/
}

Table 1. Summary of the main properties of SN 2015da and its environment.

\begin{tabular}{cc}
\hline \hline$\alpha[\mathrm{J} 2000]$ & $13^{\mathrm{h}} 52^{\mathrm{m}} 24^{\prime \prime} 11$ \\
$\delta[\mathrm{J} 2000]$ & $+39^{\circ} 41^{\prime} 28^{\prime \prime} .2$ \\
$M_{\mathrm{R}, \mathrm{peak}}$ & $-20.45 \mathrm{mag}$ \\
Host & NGC 5337 \\
Distance modulus & $33.63 \mathrm{mag}{ }^{(a)}$ \\
Galactic reddening & $E(B-V)=0.01 \mathrm{mag}(b)$ \\
Host reddening & $E(B-V)=0.97 \pm 0.27 \mathrm{mag}$ \\
Metallicity & $12+\log [\mathrm{O} / \mathrm{H}]=8.48 \mathrm{dex}$ \\
\hline
\end{tabular}

References. ${ }^{(a)}$ Tully et al. (2016); ${ }^{(b)}$ Schlafly \& Finkbeiner (2011).

The local metallicity in the environment of the H II region was inferred from the integrated flux ratios of the diagnostic lines $\mathrm{H} \alpha$ and [N II] $\lambda 6583$. Following Pettini \& Pagel (2004) and using their recalibration of the $\mathrm{N} 2 \equiv \log ([\mathrm{N}$ II $] \lambda 6583 / \mathrm{H} \alpha)$ index (Denicoló et al. 2002), we get $12+\log [\mathrm{O} / \mathrm{H}]=8.45 \mathrm{dex}$ from their linear relation, and 8.39 dex using their third-order polynomial fit (their Eqs. (1) and (2), respectively). We could not use other emission line diagnostics (such as O3N2; Alloin et al. 1979) since we did not detect the required lines for these methods (i.e., [O III] $\lambda 4363$ or $\lambda 5007)$. Since it is well established that peripheral $\mathrm{H}$ II regions have lower metallicities than those closer to the galactic centers, we then used metallicity gradients available in the literature to infer an estimate of the local oxygen abundance at the position of SN 2015da with respect to the value inferred at the position of the HII region. We computed the de-projected normalized distances $\left(r_{\mathrm{SN}} / R_{25}\right.$ and $r_{\mathrm{HII}} / R_{25}$ ) from the center of NGC 5337 following Hakobyan et al. (2009, their Eqs. (1) and (2); see also Taddia et al. 2013a). Using the coordinates of the SN and the host center, the inclination and major axis position angle (PA) reported in the HyperLeda database, we inferred de-projected distances $r_{\mathrm{SN}} / R_{25}=0.35$ and $r_{\mathrm{HII}} / R_{25}=0.47\left(17^{\prime \prime} .38\right.$ and $23^{\prime \prime}$. 65 offset from the center of NGC 5337) for the SN and the H II region, respectively. Assuming a metallicity gradient of $-0.47 R / R_{25}$ (i.e., the average of the metallicity gradients reported in Pilyugin et al. 2004, for a sample of galaxies), we extrapolated the oxygen abundance at the galactocentric distance of SN 2015da, obtaining an averaged nearly solar value of $12+\log [\mathrm{O} / \mathrm{H}]=8.48 \mathrm{dex}$ (assuming a solar metallicity of $12+\log [\mathrm{O} / \mathrm{H}]=8.69 \mathrm{dex}$; Asplund et al. 2009). Although affected by the assumption on the metallicity gradient and by the fact that line diagnostic methods are generally believed to underestimate local abundances (see, e.g., LópezSánchez et al. 2012), this result is in agreement with the one reported by Taddia et al. (2015) on a sample of interacting $\mathrm{SNe}$, showing that long-lasting SNe IIn, like SN 2015da, seem to occur in marginally subsolar metallicity environments.

The host galaxy extinction was estimated as detailed below. From the DEIMOS spectrum, we inferred an equivalent width (EW) of 1.3 and $1.2 \AA$ for the D2 and D1 lines of the Na I D doublet, respectively, which are both above the linearity range of the relation between the Na I D EW and $E(B-V)(\simeq 0.6 \AA$; see Poznanski et al. 2012). This suggests a highly extinguished environment. We therefore used different methods to estimate the local extinction. Although Type IIn SNe show a remarkable heterogeneity, we notice a strong similarity between the spectroscopic and photometric evolutions of SN 2015da and SN 2010j1 (e.g., Stoll et al. 2011; Zhang et al. 2012; Fransson et al. 2014; Borish et al. 2015, see Sect. 3). On the basis of this similarity, 


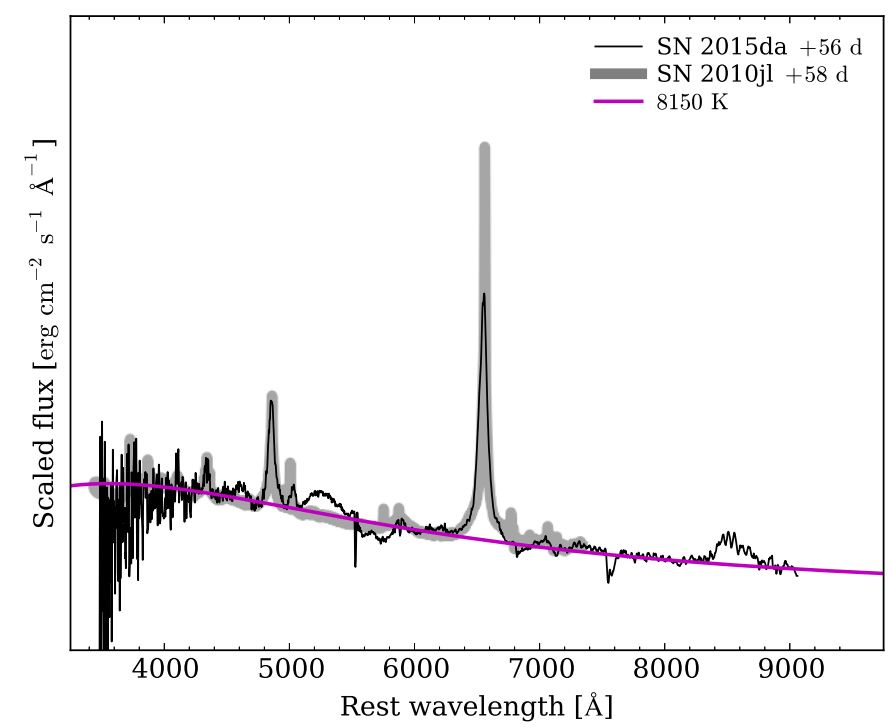

Fig. 2. Fit to the continuum of the optical spectrum obtained at $+56 \mathrm{~d}$ after $R$-band maximum compared to a spectrum of the Type IIn SN 2010jl at a similar phase. The fit was obtained by varying the amount of the extinction for SN 2015da in order to have a BB temperature comparable with that computed for SN 2010jl. We first fixed $R_{V}=3.1$ and then let it vary within the $R_{V}=2-4$ range.

we used the available data to infer an estimate of the extinction, fitting the evolution of the spectral continuum of SN 2015da to that of SN 2010jl. Since Type IIn SNe typically show prominent Balmer lines in emission, we compared the evolution of the temperature of the pseudo photosphere estimated fitting black-body (BB) functions to selected regions of the spectral continuum (i.e., those not affected by the presence of strong emission lines, like $\mathrm{H} \alpha$ or $\mathrm{H} \beta$ ). We therefore fitted the temperatures using a standard extinction law (assuming $R_{V}=3.1$; Cardelli et al. 1989) within the first $\simeq 200 \mathrm{~d}$ from the $R$-band maximum (in this context, we refer to the $R$-band maximum, since the explosion epoch of SN 2010jl is not well constrained). For each epoch, we let the $V$-band extinction vary within the $A_{V}=0-4$ mag range with steps of $0.08 \mathrm{mag}$, considering the one minimizing the $\chi^{2}$ distributions derived at each epoch as the best fit model. With this method, we obtain $E(B-V) \simeq 0.97 \pm 0.10 \mathrm{mag}$ for $R_{V}=3.1$ (assuming a conservative 10\% error due to the uncertainty in the flux calibration of the spectra), whereas we do not notice a significant improvement (although we get a slightly higher extinction) when allowing $R_{V}$ to vary within the 2-4 range (see Fig. 2). A similar approach is to consider the evolution of the spectral energy distribution (SED) computed using those bands typically unaffected by the presence of strong emission lines, which, therefore, map the shape of the spectral continuum $(B V I H K)$ more closely. Following the prescriptions of Tartaglia et al. (2018) (see also Elias-Rosa et al. 2006, for an equivalent approach), we fitted the evolution of the SED of SN 2015da to that of SN 2010jl (computed using the same bands), finding a good agreement with the previous result, obtaining $E(B-V) \simeq$ $0.92 \pm 0.10 \mathrm{mag}$.

In order to test the robustness of these estimates, we compared this value with that obtained using an alternative method, based on the presence of particular features in sufficiently highresolution spectra of highly extinguished SNe. While we do not notice any strong evidence of diffuse interstellar bands (DIBs; see, e.g., Herbig 1995; Sollerman et al. 2005) in the DEIMOS spectrum, we inferred an independent estimate of the local extinction by fitting the strong $\mathrm{Na} I \mathrm{D}$ features using the Voigt profile fitting VPFIT ${ }^{8}$ code (and $R_{V}=3.1$ ), convolving theoretical Voigt line profiles with the spectral resolution to fit the observed features, therefore obtaining an Na I column density of $\log N_{\mathrm{Na}}=13.53 \pm 0.08$ dex. Following Ferlet et al. (1985) we used the relation:

$\log N(\mathrm{Na} \mathrm{I})=1.04\left[\log N\left(\mathrm{H} \mathrm{I}+\mathrm{H}_{2}\right)\right]-9.09$,

(with $N\left(\mathrm{NaI}\right.$ ) and $N(\mathrm{H})$ in $\mathrm{cm}^{-2}$ ) to infer the neutral hydrogen column density and thus compute an estimate of the color excess through the relation given by Bohlin et al. (1978):

$\frac{N\left(\mathrm{H} \mathrm{I}+\mathrm{H}_{2}\right)}{E(B-V)}=5.8 \times 10^{21} \mathrm{~cm}^{-2} \mathrm{mag}^{-1}$.

Using these prescriptions, we get an additional color excess due to the extinction in the $\mathrm{SN}$ environment of $E(B-V)=$ $0.96 \pm 0.27 \mathrm{mag}$, which is in agreement with the estimates reported above. Therefore, in the following, we use $E(B-V) \simeq$ $0.97 \mathrm{mag}$ (assuming a standard extinction law with $R_{V}=3.1$ ) as the host galaxy reddening, implying a total color excess of $E(B-V)=0.98 \pm 0.30 \mathrm{mag}$ (accounting for the uncertainties of the different methods used above) in the direction of SN 2015da.

\section{Analysis and discussion}

In the following, we qualitatively discuss the main results of the analysis on the available photometric and spectroscopic data. Indepth modeling and focused analysis are to be the subject of a forthcoming paper.

\subsection{Photometry}

Optical, near- and mid-IR (NIR and MIR, respectively) light curves of SN 2015da are shown in Figs. 3 and 4, respectively, and the apparent magnitudes are reported in Tables A.1-A.4 (available through the CDS), along with apparent magnitudes of the local standard stars used for the photometric calibration (Table A.5). Optical and NIR photometric data were mainly obtained using the telescopes of the Las Cumbres Observatory ${ }^{9}$ network (Brown et al. 2013) within the Supernova Key Project and by the NUTS collaboration ${ }^{10}$.

To estimate the explosion epoch, we first fitted a power law to the early $R$-band light curve (the one with the best coverage of the rise time including the latest nondetection limit) at $t_{\mathrm{MJD}} \leq 57122$ (i.e. $\simeq 90 \mathrm{~d}$ after the discovery). This choice is based on the shape of the $R$-band light curve during the rise, which is well-reproduced by a power law at early phases only. Performing $10^{4}$ Monte Carlo (MC) simulations randomly shifting the data points within their errors, we find that the early $R$-band light curve of SN 2015da evolves as $L \propto(t-$ $57031.8893 \pm 0.0007)^{0.283 \pm 0.006}$, suggesting an explosion epoch roughly coincident with the discovery. On the other hand, given the large uncertainties surrounding the early points, we prefer to take the midpoint between the discovery and the last nondetection as a more conservative estimate, therefore assuming 2015 January 8.45 UT (JD $=2457030.945, \simeq 1.45 \mathrm{~d}$ before the first detection) as the explosion epoch of SN 2015da. Rise times in different bands were computed in the same way, fitting highorder polynomials to the light curves through MC simulations. We find slow rise times in all bands, increasing from the blue

\footnotetext{
8 https://www.ast.cam.ac.uk/ rfc/vpfit.html

9 https://lco.global/

10 http://csp2.lco.cl/not/
} 


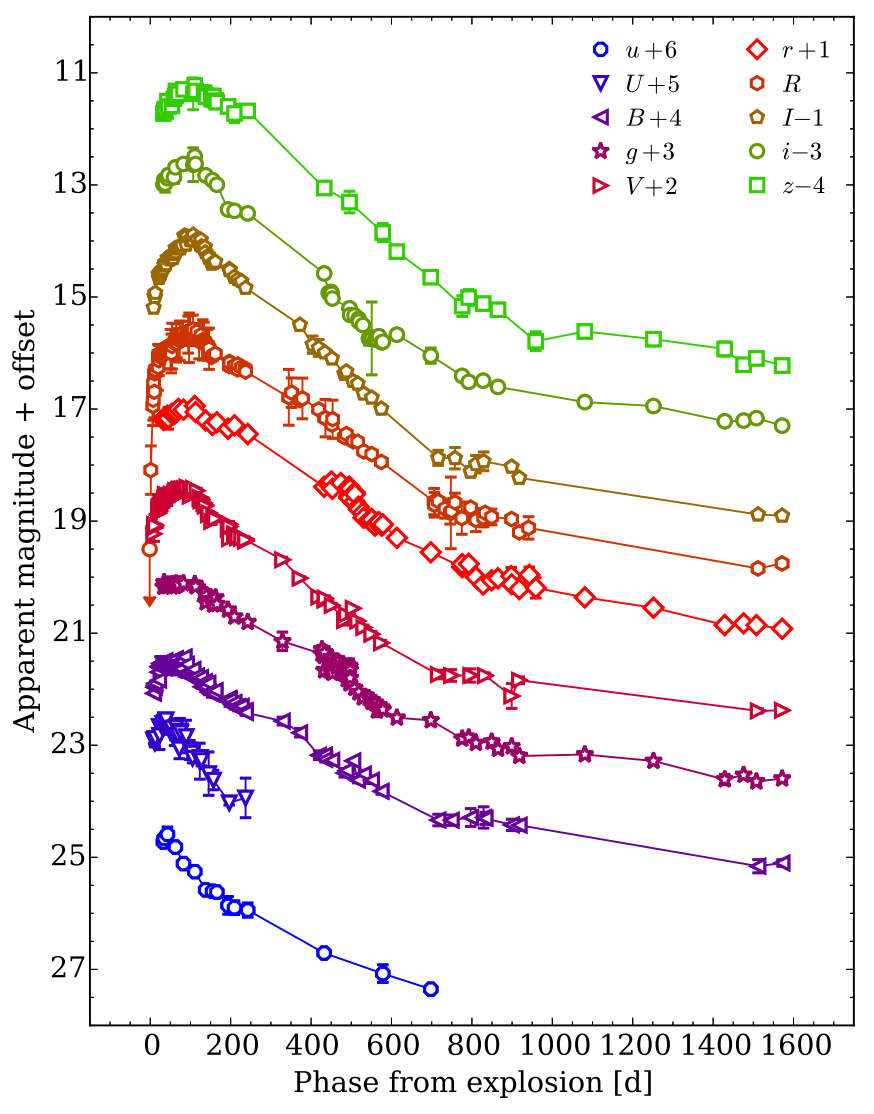

Fig. 3. Optical light curves of SN $2015 \mathrm{da}$ during the first $\simeq 4 \mathrm{yr}$ after the explosion. Magnitudes are calibrated in the Vega $(U B V R I)$ and $\mathrm{AB}$ systems (ugriz) and are not corrected for the MW or host galaxy extinction.

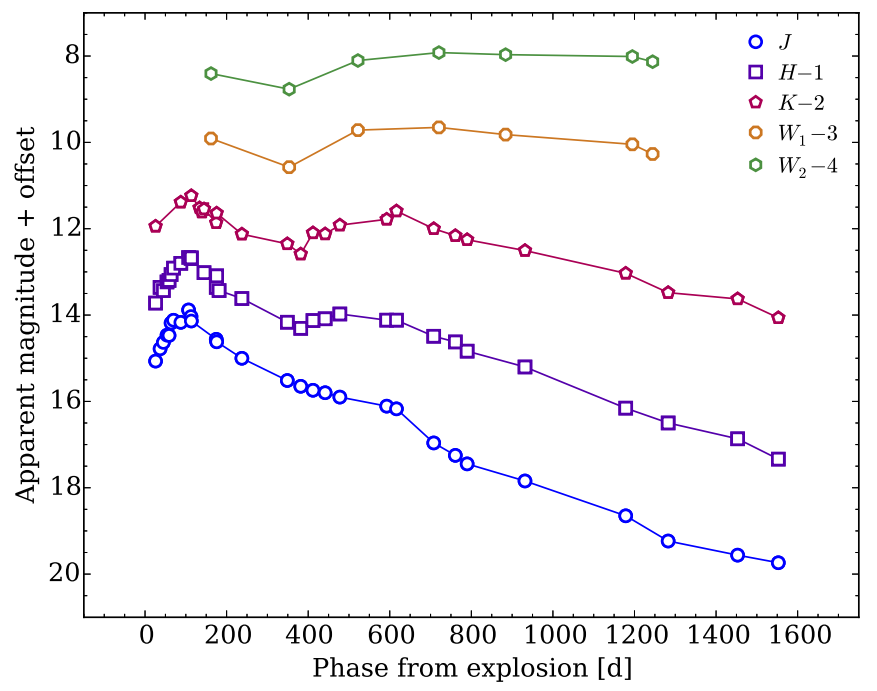

Fig. 4. IR light curves of $S N 2015$ da during the first $\simeq 4$ yr after the explosion. Magnitudes are calibrated in the Vega system.

$\left(t_{\text {rise }, \mathrm{U}}=35 \pm 10 \mathrm{~d}\right)$ to the red bands $\left(t_{\text {rise }, \mathrm{R}, \mathrm{I}}=100 \pm 5 \mathrm{~d}\right)$, with the longest period found in the NIR bands (106 \pm 10 , $110 \pm 10$, and $126 \pm 10 \mathrm{~d}$ in $J, H$, and $K$-band, respectively), resulting in bright absolute magnitudes at peak (e.g., $M_{R}=$ $-20.45 \pm 0.55$ and $M_{I}=-20.39 \pm 0.55 \mathrm{mag}$; see Table 2). Errors on the absolute magnitudes are dominated by the large uncertainty in the distance to NGC 5337. In Fig. 5, we show that the resulting $R$-band peak magnitude is consistent with that of
Table 2. Rise times and absolute peak magnitudes of SN 2015da in optical and NIR bands.

\begin{tabular}{cccc}
\hline \hline Band & $\begin{array}{c}t_{\max }-t_{\text {expl }} \\
(\mathrm{d})\end{array}$ & $\begin{array}{c}\text { Error } \\
(\mathrm{d})\end{array}$ & $\begin{array}{c}M_{\text {peak }} \\
(\mathrm{mag})\end{array}$ \\
\hline$u$ & 33 & 15 & -19.61 \\
$U$ & 35 & 10 & -20.62 \\
$B$ & 52 & 5 & -20.08 \\
$g$ & 70 & 10 & -20.18 \\
$V$ & 87 & 10 & -20.19 \\
$R$ & 100 & 3 & -20.45 \\
$i$ & 107 & 10 & -20.16 \\
$I$ & 100 & 5 & -20.39 \\
$z$ & 107 & 10 & -19.85 \\
$J$ & 106 & 6 & -20.45 \\
$H$ & 110 & 5 & -20.48 \\
$K$ & 126 & 6 & -20.74 \\
\hline
\end{tabular}

Notes. Errors on the absolute magnitudes $(\sim 0.55 \mathrm{mag}$ in all bands) are dominated by the uncertainties on the distance modulus and the extinction.

SN 2010jl (Fransson et al. 2014), also showing many other features comparable to those observed in SN 2015da (see below and Sect. 3.2).

The $R$-band light curve is the one with the best coverage thanks to the well-cadenced early observations provided by the amateurs. During the first $\sim 26 \mathrm{~d}$, it shows a relatively fast rise at a rate of $0.2 \mathrm{mag} \mathrm{d}^{-1}$, followed by a flattening in the light curve with a rate decreasing to $\simeq 0.06$ mag d $^{-1}$ in the remaining $74 \mathrm{~d}$ before maximum. Only a handful of Type IIn SNe have rises with such good coverage and well-estimated explosion epochs (see, e.g., the samples discussed in Ofek et al. 2014a and Nyholm et al. 2020). A good sampling of the peak was also obtained in $V$ and $I$, where we notice similar slow rises $\left(0.005 \mathrm{mag} \mathrm{d}^{-1}\right.$ and $0.009 \mathrm{mag} \mathrm{d}^{-1}$ ) at $+21 \lesssim t \lesssim+100 \mathrm{~d}$. After peak, the $R$ band light curve shows a first rapid decline, with the luminosity getting $\simeq 0.5$ mag fainter in $\sim 65 \mathrm{~d}$, and then it flattens at a rate of $0.004 \mathrm{mag} \mathrm{d}^{-1}$. At $\simeq+400 \mathrm{~d}$, the slope slightly increases to $0.005 \mathrm{mag} \mathrm{d}^{-1}$ until $\simeq+700 \mathrm{~d}$, when we note a further flattening, with the exception of the $U / u$ bands, where the light curves show linear declines after peak $\left(0.003 \mathrm{mag} \mathrm{d}^{-1}\right.$ and $0.008 \mathrm{mag} \mathrm{d}^{-1}$ in $u$ and $U$, respectively, where the discrepancy can be attributed to the larger scatter in the $U$-band light curve). We notice a similar behavior in the other optical bands.

The relatively bright peak magnitudes of SN 2015da are comparable to the brighter end of the distribution presented in Nyholm et al. (2020) (see also Silverman et al. 2013, for a preliminary analysis on PTF SNe IIn), extending the range of luminosities inferred by Kiewe et al. (2012) to slightly higher values. The rise times observed in SN 2015da, on the other hand, seem to fall way outside the range found for their sample of 42 Type IIn $\mathrm{SNe}$, although a similar (but still $\sim 40 \mathrm{~d}$ shorter) rise was also observed in the overluminous Type IIn SN 2006gy (Ofek et al. 2007; Smith et al. 2007; Fox et al. 2015b; Agnoletto et al. 2009, see Fig. 5, right panel). An explanation for the lack of very long-lasting SNe IIn might be the still low number of early discoveries for this type of transient, for which some progress has been made to drastically increase the quality of the data in samples, yet it might still significantly affect statistical studies.

NIR light curves evolve in a similar way up to $\simeq+400 \mathrm{~d}$, where we notice a rebrightening, more pronounced in the $K$-band, lasting $\simeq 240 \mathrm{~d}$ with an increase of $\simeq 1$ mag. In order 

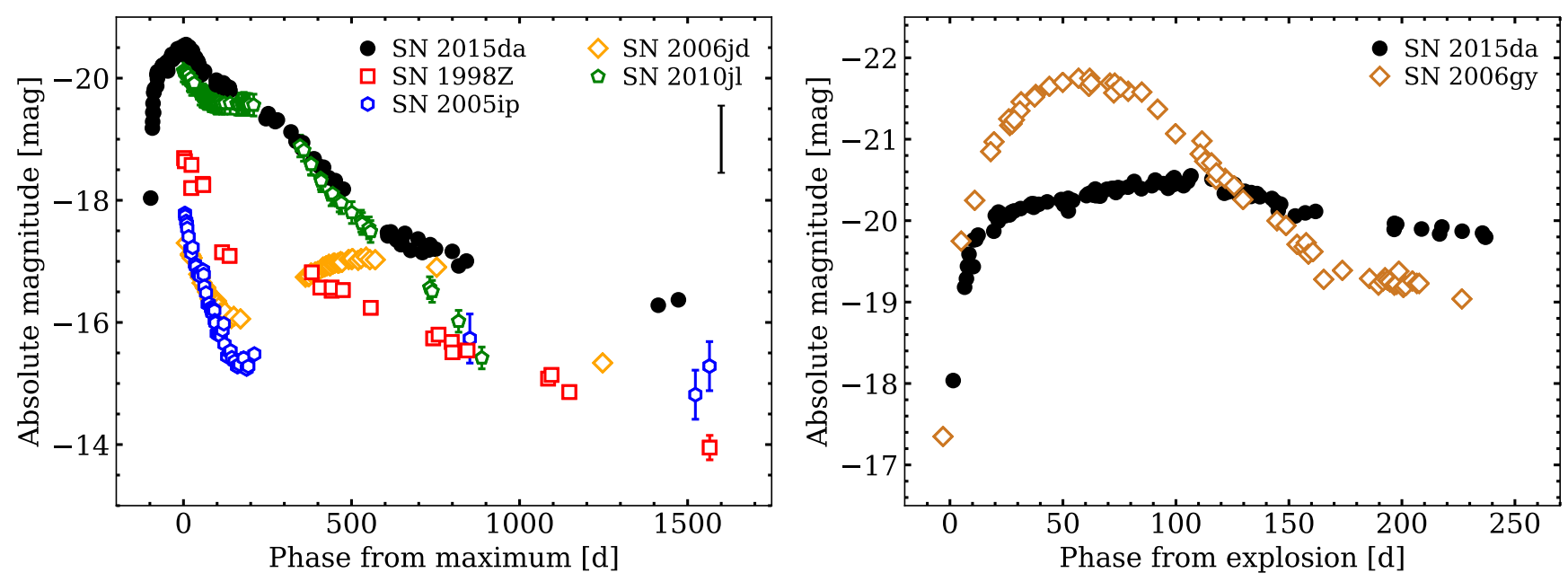

Fig. 5. Left: comparison of the $R$-band light curve of SN 2015da with those of SNe 1988Z (Turatto et al. 1993), 2005ip, 2006jl (Stritzinger et al. 2012) and 2010j1 (Fransson et al. 2014). Absolute magnitudes were obtained using Milky Way and host galaxy extinctions and adopting distances reported in the literature. The representative error bar corresponds to the uncertainty on the distance of NGC 5337. Right: comparison of the R-band light curve of SN $2015 \mathrm{da}$ with that of the overluminous SN $2006 \mathrm{gy}$ up to $\simeq 250 \mathrm{~d}$.

to collect the most complete information possible, we searched for NEOWISE Reactivation Survey detections of SN 2015da in the $W_{1}(3.4 \mu \mathrm{m})$ and $W_{2}(4.6 \mu \mathrm{m})$ bands. The first detection of a source at the SN position occured during the pass of June 2015 (18-25), $162 \mathrm{~d}$ after the explosion (see Table A.4). No detections were recorded during the previous pass occurred a few days before the SN explosion (25-31 Dec. 2014), for which we only obtained upper limits $(W 1>15.7 \mathrm{mag}$ and $W 2>15.3 \mathrm{mag})$. Since then, the SN was detected at all passings, with a cadence of approximately six months in both bands (Table A.4) showing a similar evolution with respect to the NIR bands (see Fig. 4).

\subsubsection{Evolution of the spectral energy distribution and analysis of the near infrared excess}

In order to model the excess in the IR luminosity observed at $t \gtrsim$ $400 \mathrm{~d}$, we analyzed the evolution of the SED of SN 2015da from $t=+33 \mathrm{~d}$ to $+1233 \mathrm{~d}$ with a regular interval of $50 \mathrm{~d}$. This choice is based on the available photometric points (e.g., the first available $u$-band point is at $+33 \mathrm{~d}$ ). Apparent magnitudes were interpolated at each epochs, corrected for the total extinction as estimated in Sect. 2, and converted in flux using the appropriate effective wavelengths and zero points available in the literature for each band. Effective wavelengths were corrected for the heliocentric recessional velocity inferred from the narrow Na I D features in absorption (see Sect. 3.2). Figure 6 shows the results of the fit at selected epochs, reported in Table 3.

We used BB functions (using a single, or a combination of two BBs, when appropriate) to fit the SED evolution, excluding $u$-band fluxes at $t \geq+83 \mathrm{~d}$ and bands bluer than $R$ at $t \geq+183 \mathrm{~d}$, since strong blends of Fe II lines start to dominate the blue spectral continuum around this epoch, and the SED can no longer be approximated by a BB at $\lambda \lesssim 5500 \AA$. On the other hand, we estimated the contributions of $\mathrm{H} \alpha$ and $\mathrm{Pa} \gamma$ lines in order to remove them from the integrated fluxes in the $r, R$ and $J$ bands. Since the integrated flux of Pa $\gamma$ could be directly estimated from the spectra only at two epochs (i.e., $+26 \mathrm{~d}$ and $+606 \mathrm{~d}$ ), we linearly interpolated its contribution to the $J$-band at the desired epochs, while the evolution of $\mathrm{H} \alpha$ was estimated with higher accuracy (see Sect. 3.2.2). Similarly, to avoid extrapolation, we did not include MIR fluxes at $t<+183 \mathrm{~d}$.
The SEDs were modeled following Suzuki \& Fukugita (2018, see their Eqs. (1) and (2)) through a Markov chain Monte Carlo $\left(\mathrm{MCMC}^{11}\right)$ routine based on the PYTHON EMCEE package, using the following expression:

$f_{\lambda}(R, T)=\frac{2 \pi h c^{2}}{D^{2} \lambda^{5}}\left(\frac{R_{\mathrm{h}}^{2}}{e^{h c / \lambda k_{\mathrm{B}} T_{\mathrm{h}}}-1}+\frac{R_{\mathrm{w}}^{2}}{e^{h c / \lambda k_{\mathrm{B}} T_{\mathrm{w}}}-1}\right)$,

where $R_{\mathrm{h}}, T_{\mathrm{h}}, R_{\mathrm{w}}$, and $T_{\mathrm{w}}$ are the radii and temperatures of the hot and warm components, respectively, and $D$ is the distance to SN 2015da. Adopting a luminosity distance of $53.2 \pm 13.2 \mathrm{Mpc}$ (see Sects. 1 and 2), we could therefore infer the radii of the two emitting regions, assuming pure thermal emission at the equilibrium temperatures $T_{\mathrm{h}}$ and $T_{\mathrm{w}}$ and spherical symmetry. A more detailed analysis, including geometrical considerations (such as the one performed by Andrews et al. 2011, for SN 2010j1), is beyond the scope of this paper and is to be presented in a forthcoming paper. Up to $+433 \mathrm{~d}$, the SED is well-reproduced using a single $\mathrm{BB}$ with a temperature decreasing from $12700 \pm 150 \mathrm{~K}$ to $5850 \pm 90 \mathrm{~K}$. During the same period, the radius of the photospheric component increases from $(1.81 \pm 0.02) \times 10^{15}$ to $(3.49 \pm 0.07) \times 10^{15} \mathrm{~cm}$ in the first $150 \mathrm{~d}$, and then decreases during the following $500 \mathrm{~d}$ (see Table 3 ).

From $+433 \mathrm{~d}$, we need a second $\mathrm{BB}$ to properly reproduce the SED, showing a slightly decreasing temperature (after an initial increase from $1280 \pm 40 \mathrm{~K}$ to $1510 \pm 20 \mathrm{~K}$, probably due to a residual contamination from the photospheric component) and a BB radius increasing from $(2.06 \pm 0.10) \times 10^{16}$ to $(2.60 \pm 0.05) \times 10^{16} \mathrm{~cm}$. At later times $(t \geq+733 \mathrm{~d})$, the fit to the IR flux is well-reproduced by a single warm component with slightly decreasing temperatures (from 1400 to $1000 \mathrm{~K}$, see Table 3). This suggests a negligible contribution of the photospheric component in the IR domain and the absence of an additional cooler component at these epochs.

IR excesses are more common in long-lasting Type IIn SNe at late times $(t \gtrsim 100 \mathrm{~d})$ than in other SN types (e.g., Fox et al. 2011) and are generally interpreted as thermal emission from either preexisting or newly formed dust (see, e.g., Gerardy et al. 2002; Mattila et al. 2008). To get a rough estimate of the mass of dust needed to produce the observed excess, we followed the

${ }^{11} \mathrm{http}: / / \mathrm{dfm}$. io/emcee/current/ 

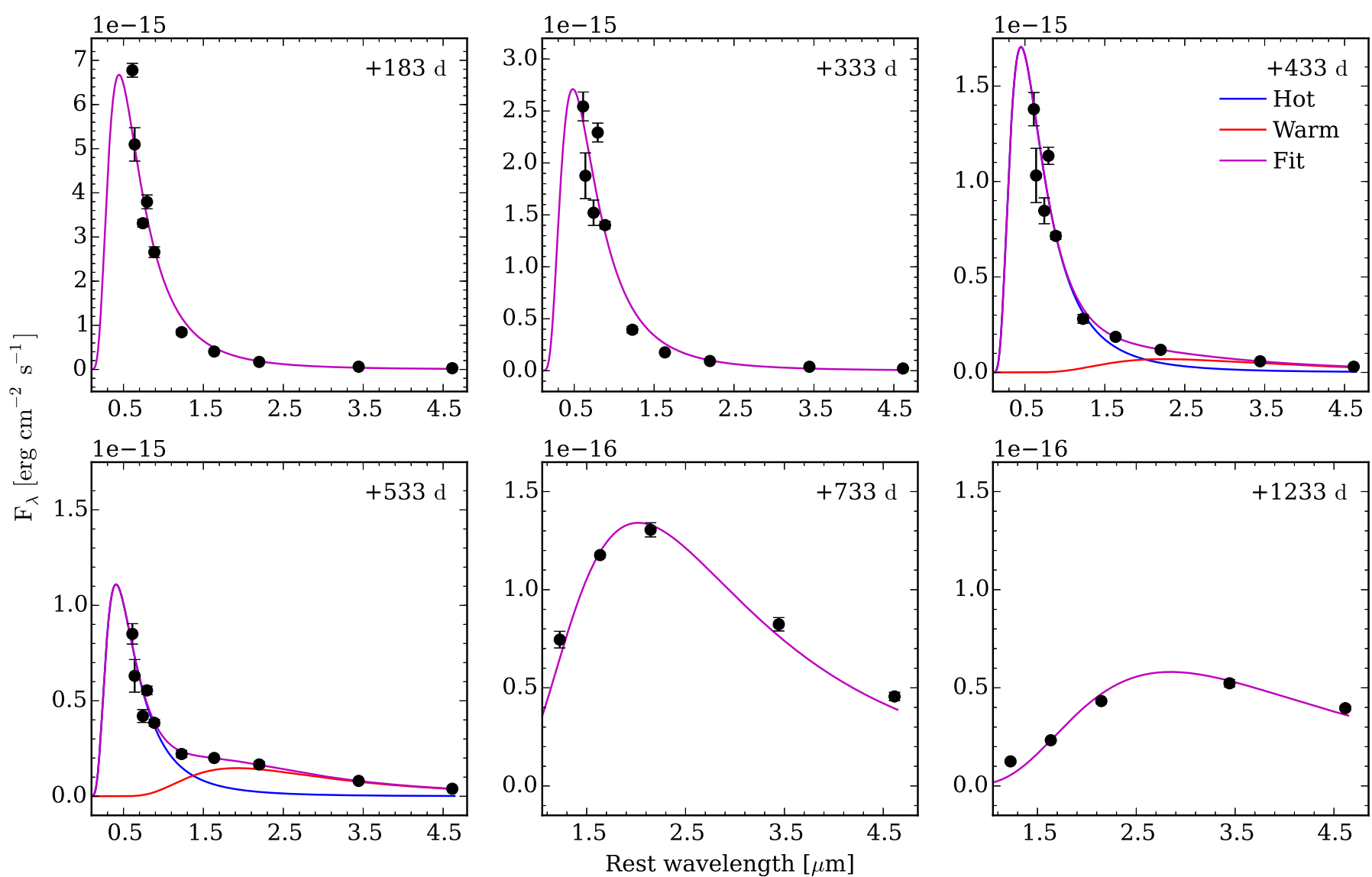

Fig. 6. Fit of a hot and a warm BB component to the evolution of the SED of SN 2015da. A warm component dominating at NIR wavelengths is clearly visible from $t \gtrsim+430 \mathrm{~d}$, increasing its strength with time. At $t \gtrsim+730 \mathrm{~d}$ a single BB is sufficient to reproduce the SED at IR wavelengths $(\lambda \gtrsim 1.2 \mu \mathrm{m})$.

prescriptions of Fox et al. (2010), fitting the following expression to the observed SEDs:

$f(\lambda)=B_{\lambda}\left(T_{\mathrm{h}}\right)+\frac{M_{\text {dust }} B_{\lambda}\left(T_{\text {dust }}\right) \kappa_{\lambda}(a)}{D^{2}}$,

where $B_{\lambda}\left(T_{\mathrm{h}}\right)$ is the $\mathrm{BB}$ resulting from the hot component (from Eq. (3)), $M_{\text {dust }}$ is the total mass of the dust, $B_{\lambda}\left(T_{\text {dust }}\right)$ the BB at the temperature of the dust, $T_{\text {dust }}, \kappa_{\lambda}(a)$ the mass absorption coefficient for a dust particle of radius $a$ as derived from Mie theory, and $D$ is the distance of the emitting source to the observer. This formalism assumes only thermal emission at the equilibrium temperature $T_{\mathrm{d}}$ and dust composed entirely either of carbon- or oxygen-rich grains (graphite and silicates, respectively) of a single size. Following Stritzinger et al. (2012), we fitted the model to the observed SEDs using dust grains of three sizes $(0.01,0.1$, and $1 \mu \mathrm{m}$, see Table 4$)$, fixing $T_{\mathrm{h}}, R_{\mathrm{h}}$ to the values derived from Eq. (3) (Table 3). For $s=0.1 \mu \mathrm{m}$, at $+1233 \mathrm{~d}$ we get $\simeq 5$ and $10 \times 10^{-3} M_{\odot}$ of preexisting dust in the CSM for graphite and silicates, respectively. As a comparison, for the same size of graphite grains, Fox et al. (2013) found masses in the $2 \times 10^{-5}-3 \times 10^{-2} M_{\odot}$ range for their sample of SNe IIn with available Spitzer data, so the masses inferred using Eq. (4) for SN 2015da are compatible with their derived values. Assuming a standard gas-to-dust ratio of $1: 100$, this indicates a CSM mass of $0.5-1 M_{\odot}$ for SN $2015 \mathrm{da}$, which is similar to that inferred by Fox et al. (2013) for SN 2010jl (1-2 $M_{\odot}$ taking the gas-to-dust ratio computed by Mauron \& Josselin 2011). On the other hand, in Sect. 3.1.2 we find a much higher value of swept up CSM $\left(\simeq 8 M_{\odot}\right)$. This difference could be due to the assumptions made when estimating the dust mass (such as grain sizes), composition, and geometrical configuration. More accurate models are necessary to infer the physical properties of the dust producing the IR excess observed in SN 2015da.

We note that $\mathrm{BB}$ radii inferred for the IR component (Table 3 ) are similar to those derived for the dust evaporation radius in SN 2010jl by Fransson et al. (2014) for large graphite grains. This would seem to favor large graphite grains as the main components of the preexisting dust. On the other hand, since we do not have access to MIR spectra, we cannot rule out silicates or graphite as the main composition for the dust grains. The highest $T_{\mathrm{d}}$ we infer fitting Eq. (3) $(\simeq 1510 \mathrm{~K}$ at $+533 \mathrm{~d}$, see Table 3$)$ is roughly coincident with the typical evaporation temperature of silicates (1500 K; see, e.g., Draine \& Lee 1984), although at those epochs, the photospheric component might still contribute to the IR SED, and, at later times, the temperature decreases well below the evaporation value for both types of grains. In addition, we do not have measurements at $\lambda>4.6 \mu \mathrm{m}$, the region where graphite and silicates show the most divergent behavior in their emission efficiencies (see, e.g., Fig. 4 in Fox et al. 2010).

We therefore derived the dust mass from Eq. (4), using $\kappa_{\lambda}(a)$ for both graphite and silicates as given in Draine \& Lee (1984) and Laor \& Draine (1993). From the results reported in Table 4, we note that graphite grains with a radius of $1 \mu \mathrm{m}$ give the closest $T_{\mathrm{d}}$ values with respect to those inferred fitting Eq. (3) to the SED evolution (see Table 3), although comparable values are also obtained for smaller silicate grains $(a=0.01 \mu \mathrm{m})$. The discrepancies among the results obtained using the two models might be a result of the assumptions on the geometrical distribution, 
Table 3. Main parameters of the BB fit to the observed SED evolution of SN 2015da.

\begin{tabular}{cccccc}
\hline \hline $\begin{array}{c}\text { Phase } \\
(\mathrm{d})\end{array}$ & $\begin{array}{c}T_{\mathrm{h}}(\mathrm{err}) \\
(\mathrm{K})\end{array}$ & $\begin{array}{c}R_{\mathrm{h}}(\mathrm{err}) \\
\left(10^{15} \mathrm{~cm}\right)\end{array}$ & $\begin{array}{c}T_{\mathrm{W}}(\mathrm{err}) \\
(\mathrm{K})\end{array}$ & $\begin{array}{c}R_{\mathrm{W}}(\mathrm{err}) \\
\left(10^{16} \mathrm{~cm}\right)\end{array}$ & $\begin{array}{c}\log L_{\mathrm{NIR}}(\mathrm{err}) \\
\left(\mathrm{erg} \mathrm{s}^{-1}\right)\end{array}$ \\
\hline+33 & $12700(150)$ & $1.81(0.02)$ & - & - & - \\
+83 & $9650(110)$ & $2.84(0.04)$ & - & - & - \\
+133 & $8280(180)$ & $2.93(0.08)$ & - & - & - \\
+183 & $6500(90)$ & $3.49(0.07)$ & - & - & - \\
+233 & $6820(90)$ & $3.01(0.05)$ & - & - & - \\
+283 & $6410(100)$ & $2.85(0.06)$ & - & - & - \\
+333 & $5990(100)$ & $2.74(0.06)$ & - & - & - \\
+383 & $5850(90)$ & $2.42(0.05)$ & - & - & - \\
+433 & $6450(260)$ & $1.81(0.11)$ & $1280(40)$ & $2.06(0.10)$ & $41.91(0.07)$ \\
+483 & $6420(330)$ & $1.56(0.12)$ & $1430(30)$ & $1.98(0.07)$ & $42.07(0.05)$ \\
+533 & $7300(520)$ & $1.10(0.11)$ & $1510(20)$ & $2.01(0.06)$ & $42.18(0.03)$ \\
+583 & $8260(710)$ & $0.81(0.09)$ & $1490(20)$ & $2.13(0.05)$ & $42.20(0.03)$ \\
+633 & $8060(660)$ & $0.74(0.08)$ & $1430(10)$ & $2.30(0.05)$ & $42.20(0.02)$ \\
+683 & $12620(1890)$ & $0.41(0.06)$ & $1400(10)$ & $2.41(0.05)$ & $42.20(0.02)$ \\
+733 & - & - & $1400(10)$ & $2.32(0.05)$ & $42.17(0.02)$ \\
+783 & - & - & $1340(10)$ & $2.42(0.05)$ & $42.13(0.02)$ \\
+833 & - & - & $1300(10)$ & $2.50(0.05)$ & $42.11(0.02)$ \\
+883 & - & - & $1250(10)$ & $2.60(0.05)$ & $42.07(0.02)$ \\
+933 & - & - & $1190(5)$ & $2.89(0.02)$ & $42.07(0.02)$ \\
+983 & - & - & $1153(5)$ & $2.97(0.02)$ & $42.04(0.02)$ \\
+1033 & - & - & $1120(5)$ & $3.05(0.02)$ & $42.02(0.02)$ \\
+1083 & - & - & $1089(5)$ & $3.14(0.03)$ & $41.99(0.02)$ \\
+1133 & - & - & $1058(5)$ & $3.25(0.02)$ & $41.97(0.02)$ \\
+1183 & - & - & $1043(5)$ & $3.21(0.05)$ & $41.93(0.04)$ \\
+1233 & - & - & $1011(5)$ & $3.39(0.05)$ & $41.93(0.03)$ \\
\hline & & & & &
\end{tabular}

Notes. Phases refer to the epoch of the explosion.

composition and grain sizes, as well as the dust covering factor (a covering factor $f<1$ would give larger radii), which do not allow us to favor carbon- or oxygen-rich grains as the main components of the dust shell.

The analysis of the IR luminosity evolution may give additional information about the nature of the dust grains responsible for the excess of radiation observed at $t \geq 433 \mathrm{~d}$. IR bolometric luminosities were computed using the $R_{\mathrm{w}}$ and $T_{\mathrm{w}}$ obtained fitting Eq. (3) to the observed SEDs (Table 3). The resulting light curve shows a slow rise during the first $100 \mathrm{~d}$ (after $+433 \mathrm{~d}$, the onset of the IR excess), a peak at $\simeq 1.6 \times 10^{42} \mathrm{erg} \mathrm{s}^{-1}$, and subsequently a settling onto a relatively long "plateau". At $+1233 \mathrm{~d}$, the last epoch at which we could interpolate available NIR and MIR photometry, we inferred a luminosity of $\simeq 8.5 \times 10^{41} \mathrm{erg} \mathrm{s}^{-1}$, suggesting a very slow decline after peak (see Table 3 ).

According to the discussion on SN 2010jl (see, e.g., Andrews et al. 2011; Smith et al. 2012; Fransson et al. 2014; Gall et al. 2014), dust responsible for the IR excess can originate from different mechanisms, including rapid formation of large dust grains in the post-shocked regions at the interface between forward and reverse shock, heating and evaporation of preexisting dust by the SN shock, or an "echo" from a preexisting outer dust shell. While emission from relatively large dust grains $(\simeq 1 \mu \mathrm{m})$ is able to reproduce the temperature of the dust inferred from broad-band photometry (see Tables 3 and 4), in the following, we show that an IR echo is the most plausible interpretation for the observed late excess in the IR luminosity of SN 2015da.

Although the formation and survival of dust grains in postshocked regions behind a radiative shock proved to be a viable explanation for the IR excess observed in a few Type IIn SNe (see, e.g., the cases of SN 2005ip and 2006jd; Stritzinger et al. 2012), Fransson et al. (2014) showed that emission from newly formed dust would not be sufficient to explain the large IR luminosity observed at late time in SN 2010jl, which is comparable to that observed in SN 2015da. In addition, condensation of dust either within the $\mathrm{SN}$ ejecta or in post-shocked regions is expected to scatter and absorb light emitted by the underlying onrushing ejecta, causing the attenuation of the red wings of the most prominent lines (e.g., $\mathrm{H} \alpha$ ) and steeper decline rates, in particular in the bluer optical light curves. We do not see such features in SN 2015da: $\mathrm{H} \alpha$ always shows symmetric profiles with respect to its centroid, and the optical light curves do not show steeper declines. We therefore do not consider newly formed dust in the CDS of SN 2015da as a plausible interpretation for the IR excess observed at $t \geq+433 \mathrm{~d}$.

A useful probe to infer the origin of the dust emission is to compare the $\mathrm{BB}$ radius of the dust component obtained from Eq. (3) to the radius of the shocked region inferred from the maximum velocity of the shock, as estimated in Sect. 3.1.2 $\left(R_{\mathrm{s}} \simeq V_{\mathrm{s}} t\right.$, with $\left.V_{\mathrm{s}} \simeq 3000 \mathrm{~km} \mathrm{~s}^{-1}\right)$, that is $\simeq 1.1 \times 10^{15} \mathrm{~cm}$ at $+433 \mathrm{~d}$. This value is a factor of two smaller than that inferred for $R_{\mathrm{w}}$ (see Table 3). For the shock to reach the dust shell at $+433 \mathrm{~d}$, we would therefore need significantly higher values for $V_{\mathrm{s}}$. We stress, however, that the value of the dust shell radius has to be considered a lower limit, since it is computed assuming spherical, symmetric geometrical configurations, and a dust covering factor $f=1$.

Another useful quantity is the evaporation radius for a given grain size, composition and bolometric luminosity at peak, namely the radius of the dust-free cavity produced by an SN outburst around the progenitor star. According to Fransson et al. (2014), an SN outburst with a peak luminosity of $10^{43} \mathrm{erg} \mathrm{s}^{-1}$ produces a dust-free cavity with a radius $\gtrsim 3.5 \times 10^{16} \mathrm{~cm}$, depending on the dust composition, the grain size, and the effective temperature of the SN, with the lower limit reached for $T_{\mathrm{SN}}=6000 \mathrm{~K}$ and carbon-rich dust grains with $a=1 \mu \mathrm{m}$. The radius of the dust-free cavity is larger than the BB radii inferred from the IR SEDs of SN 2015da at all epochs, although, as discussed above, these have to be considered as lower limits. The evaporation radius, on the other hand, also depends on the assumed evaporation temperature, which may be lower than the typical value for graphite/silicates if a more luminous outburst precedes the SN explosion, leading to a larger radius of the dust-free cavity (Dwek 1983).

These considerations lead us to the conclusion that collisional heating and evaporation of preexisting dust is not likely to be the mechanism responsible for the IR excess in SN 2015da, and that an IR echo from heated dust is a more promising alternative. A simple approach to modeling IR echoes from preexisting radiatively heated dust is to consider a spherically symmetric shell around the SN composed solely of spherical grains of a single size and composition, optically thin (at IR wavelengths) to the SN radiation (see Dwek 1983; Graham \& Meikle 1986). The observed IR radiation therefore arises from a paraboloidal surface and is significantly delayed with respect to the SN light due to light-travel time effects. In this context, the observed dust temperature is a function of the angle between the vector radius from the $\mathrm{SN}$ to the emitting shell element and the line of sight (i.e., shell elements closer to the $\mathrm{SN}$ are observed at higher temperatures) as well as the distance to the $\mathrm{SN}$ and the time from explosion.

One piece of evidence favoring this interpretation is the long plateau and very slow decline after peak observed in the IR bolometric luminosity of SN 2015da. The IR light curve of an SN 
Table 4. Mass and temperature evolution of the dust shell for grains of different composition (graphite/silicates) and sizes.

\begin{tabular}{|c|c|c|c|c|c|c|}
\hline $\begin{array}{l}\text { Phase } \\
\text { (d) }\end{array}$ & $\begin{array}{c}M_{\text {dust }}(\mathrm{err}) \\
\left(\times 10^{-3} M_{\odot}\right)\end{array}$ & $\begin{array}{c}T_{\text {dust }} \text { (err) } \\
(\mathrm{K})\end{array}$ & $\begin{array}{c}M_{\text {dust }}(\mathrm{err}) \\
\left(\times 10^{-3} M_{\odot}\right)\end{array}$ & $\begin{array}{c}T_{\text {dust }} \text { (err) } \\
\text { (K) }\end{array}$ & $\begin{array}{c}M_{\text {dust }} \text { (err) } \\
\left(\times 10^{-3} M_{\odot}\right)\end{array}$ & $\begin{array}{c}T_{\mathrm{d}}(\mathrm{err}) \\
(\mathrm{K})\end{array}$ \\
\hline & \multicolumn{2}{|c|}{$a=0.01 \mu \mathrm{m}$} & \multicolumn{2}{|c|}{$a=0.1 \mu \mathrm{m}$} & \multicolumn{2}{|c|}{$a=1 \mu \mathrm{m}$} \\
\hline+433 & $2.43(0.25) / 3.78(0.37)$ & $930(20) / 1100(25)$ & $2.25(0.23) / 3.95(0.38)$ & $875(20) / 1080(25)$ & $0.42(0.04) / 2.14(0.22)$ & $1225(30) / 1025(25)$ \\
\hline+483 & $2.13(0.16) / 3.43(0.25)$ & $20(15) / 1215$ & $7(0.15) / 3.60(0.27)$ & 9 & $8(0.03) / 1.84(0.14)$ & \\
\hline+533 & $2.16(0.14) / 3.52(0.23)$ & 10650 & $10 \times 127$ & & & $5(15)$ \\
\hline+583 & $2.41(0.15) / 3.92(0.2$ & & $2.23(0.14) / 4.12(0$. & & 3) & $1430(20$ \\
\hline+633 & $2.65(0.15) / 4.51(0.25)$ & & $2.46(0.14) /$ & & & \\
\hline+683 & $2.82(0.15) / 4.88(0.26)$ & 1020( & $0.14) / 5.13(0.27)$ & & $6(0.14)$ & $0(10)$ \\
\hline+733 & $2.27(0.13) / 4.36(0.23)$ & $1040(10) / 1$ & $2.09(0.12) / 4.61(0.24)$ & (10) 1 & $050(002$ & 140(10) \\
\hline+783 & $2.47(0.14) / 4.72(0.2$ & 1010(10)/ & $2.28(0.13) / 4.99(0$. & & $=4(002) / 241(0$ & 1300 \\
\hline+833 & $2.67(0.15) / 5.08(0.26)$ & 98 & 2.4 & 9) & 14) & $1260(1$ \\
\hline+883 & $2.90(0.16) / 5.50(0.29)$ & $955(10) / 1100(10)$ & $2.67(0.15) / 5.80(0.30)$ & $900(10) / 1080$ & $.83(0.15)$ & $1220(10) / 1045(10)$ \\
\hline+933 & $4.83(0.88) / 7.32(0.13)$ & $890(10) / 1040(10)$ & $4.47(0.81) / 7.64(0.14)$ & $840(10) / 1020(10)$ & $0.81(0.01) / 4.60(0.85)$ & $1150(10) / 965(10)$ \\
\hline+983 & $5.06(0.93) / 7.69(0.14)$ & & & & & \\
\hline+1033 & $5.31(0.97) / 8.09(0.15)$ & & & & & $1090(10)$ \\
\hline+1083 & $5.60(0.10) / 8.56(0.16)$ & $830(10) / 96$ & $5.17(0.94) / 8.92(0.16)$ & $790(10)$ & $0.96(0.02) / 5.34(0.98)$ & 1060(10)/900(10) \\
\hline+1133 & $5.99(0.11) / 9.17(0.17)$ & $815(10) / 940(10)$ & $5.52(0.10) / 9.56(0.17)$ & $775(10) / 925(10)$ & $0.10(0.02) / 5.71(0.10)$ & 1030(10)/880(10) \\
\hline+118 & $4.80(0.20) / 8.46(0.34$ & $820(10) / 930($ & $4.42(0.18) / 8.88(0.3$ & $780(10) / 920$ & $0.97(0.04) / 4.57(0.19)$ & $1020(10) / 890(10)$ \\
\hline+1233 & $5.36(0.22) / 9.47(0.38)$ & $800(10) / 910(10)$ & $4.93(0.20) / 9.93(0.39)$ & $760(10) / 895(10)$ & $0.11(0.04) / 5.15(0.21)$ & $990(10) / 865(10)$ \\
\hline
\end{tabular}

Notes. Phases refer to the estimated epoch of the explosion.

embedded in a dusty circumstellar shell depends on the chemical composition of the dust grains and the size of the dust-free cavity produced by the explosion. Wright (1980) proposed an analytical solution for the bolometric light curve of a IR light echo showing a "flat top" lasting $\sim 2 * R_{\mathrm{ev}} / c$, where $R_{\mathrm{ev}}$ is the radius up to which dust is vaporized by the $\mathrm{SN}$ explosion. Assuming a plateau length of $200 \mathrm{~d}$, the dust-free cavity produced by SN 2015da is $\simeq 2.6 \times 10^{14} \mathrm{~cm}$. Following the same prescriptions, Dwek (1985) showed that the IR luminosity produced by a carbon-rich shell is expected to be brighter and shorter in time with respect to an oxygen-rich one (see his Fig. 1). In this context, the IR light curve observed in SN 2015da would be explained by an extended shell of dust, mainly composed of silicates.

Despite the two different methods used to compute luminosities, we compared the total radiated energies obtained integrating the photospheric part of the pseudo-bolometric light curve up to $+433 \mathrm{~d}\left(9.1 \times 10^{50} \mathrm{erg}\right)$ and the IR bolometric light curve up to $+1233 \mathrm{~d}$ estimated by the BB fit $\left(8.5 \times 10^{49} \mathrm{erg}\right)$. While up to $+1233 \mathrm{~d}$, we still do not see a clear fall from the plateau in the IR light curve: the fact that only $\sim 10 \%$ of the integrated IR luminosity is reprocessed by the dust suggests that it is confined in a preexisting optically thin shell.

As the NIR and MIR follow-up campaigns of SN 2015da are still ongoing, it is likely that further photometric epochs at these wavelengths, as well as additional observations at $\lambda>4.6 \mu \mathrm{m}$, could help to unveil the nature of the IR excess.

\subsubsection{Evolution of the pseudo-bolometric light curve}

The evolution of the bolometric luminosity of SN 2015da was computed from the available photometric data in Tartaglia et al. (2016), integrating the observed SEDs without considering those regions not covered by the observations, hence only from the $u$ to the $W_{2}$ bands. The resulting "pseudo-bolometric" light curve shows a slow rise $(\simeq 100 \mathrm{~d})$ with a bright peak luminosity $\simeq 3.0 \times 10^{43} \mathrm{erg} \mathrm{s}^{-1}$. However, the early SN bolometric light curves can be significantly underestimated without taking into account the UV contribution (see also the discussion in
Tomasella et al. 2018). We therefore took the temperatures obtained fitting Eq. (3) to the observed SEDs to infer the scaling factor to account for the lack of observations at $\lambda<3500 \AA$, assuming a blackbody form. Due to the strong contamination of Fe II lines at wavelengths bluer than $\simeq 5000 \AA$ (see Sects. 3.2 and 3.1.1), at $t>400 \mathrm{~d}$ we fixed the temperature of the pseudo continuum to $5850 \mathrm{~K}$, although we note that the correction at these epochs is small (less than $10 \%$ ). We note, however, that this might still underestimate the UV contribution at early epochs, as shown by Dessart et al. (2015, their Fig. 13).

The resulting bolometric light curve, shown in Fig. 7, has a significantly shorter rise of $\simeq 30 \mathrm{~d}$, with a much brighter peak, $\simeq 6.2 \times 10^{43} \mathrm{erg} \mathrm{s}^{-1}$, more than a factor two brighter than our previous estimate, and more than three times higher than that inferred for SN 2010jl by Fransson et al. (2014). The UV contribution for SN 2010jl was, however, estimated from direct observations and the explosion is uncertain, hence its peak may have been missed, resulting in a possible brighter luminosity. In any case, this would make SN 2015da one of the brightest Type IIn SNe ever observed. The total radiated energy in the first $1233 \mathrm{~d}$, after removing the IR contribution (likely due to a dust echo; see Sect. 3.1.1), is $\simeq 10^{51} \mathrm{erg}$. This high value and the prominent narrow emission lines suggest that an ejecta-CSM interaction with a highly efficient conversion of kinetic energy into radiation is the main mechanism powering the light curve of SN 2015da (see, e.g., Fransson et al. 2014).

The luminosity evolution of SN 2015da can then be modeled in the context of an SN explosion within an extended and dense CSM (see Chevalier \& Irwin 2011; Svirski et al. 2012; Ofek et al. 2014b). After CC, a radiation-mediated shock wave propagates outwards until it breaks through the stellar photosphere. If the progenitor is surrounded by a dense and extended CSM with an optical depth $\tau_{\text {CSM }}>c / V_{\text {sh }}=\tau_{\text {sh }}$ (where $c$ is the speed of light and $V_{\text {sh }}$ the velocity of the shock), the SN shock propagates into the CSM. As a consequence, the shock breakout signal is delayed, and the reverse-forward shock structure typical of interacting transients forms at the interface between the SN ejecta and the CSM (Chevalier \& Fransson 1994). As the photon 


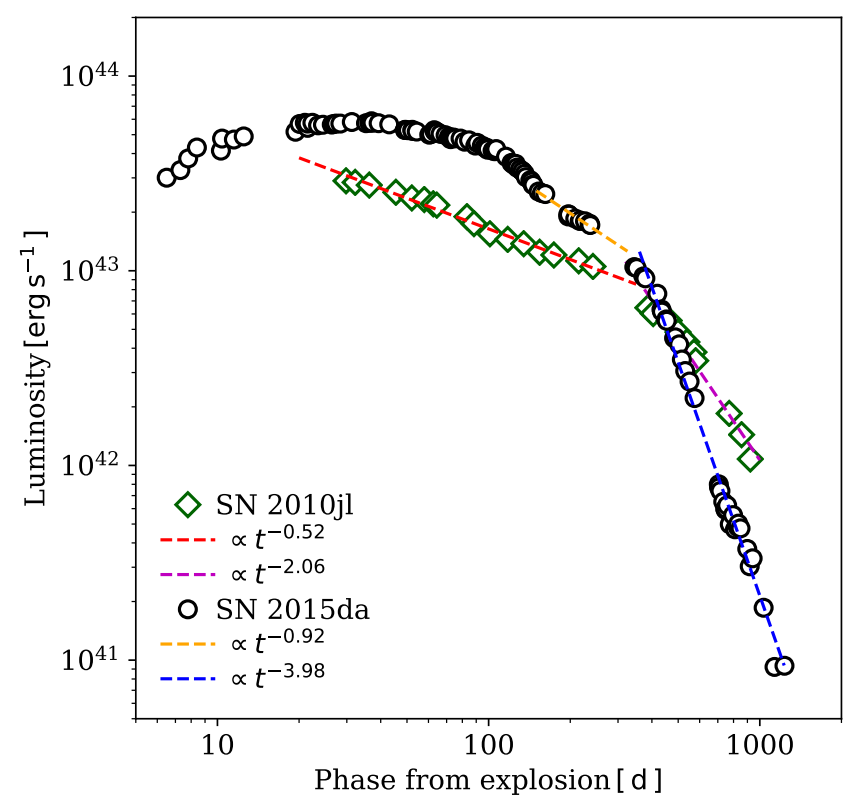

Fig. 7. Bolometric light curve of SN 2015da compared to that of SN 2010jl in logarithmic units. The best fit parameters for $+150 \leq t \leq$ $+290 \mathrm{~d}$ and $t>+290 \mathrm{~d}(+30 \leq t \leq+320 \mathrm{~d}$ and $t>+320 \mathrm{~d}$ for SN 2010jl following Fransson et al. 2014 and Ofek et al. 2014b) are reported in the legend.

diffusion time drops below the shock expansion timescale (i.e., when $\tau_{\mathrm{CSM}} \simeq \tau_{\mathrm{sh}} \simeq c / V_{\mathrm{s}}$ ), the thermalized shock radiation escapes the CSM, releasing energy over timescales that may be much longer than those typically observed in windless breakouts (see Ofek et al. 2010; Chevalier \& Irwin 2011). If the density of the CSM above the breakout layer is sufficiently high, the shock becomes collisionless, the photons are no longer trapped (Katz et al. 2012), and the kinetic energy of the ejecta is efficiently converted into radiation at a rate of $\epsilon\left(\rho_{\mathrm{CSM}} V_{\mathrm{sh}}^{2} / 2\right)\left(4 \pi r_{\mathrm{sh}}^{2} V_{\mathrm{sh}}\right)$, where $\epsilon$ is the conversion efficiency, $\rho_{\mathrm{CSM}}$ the density of the CSM, and $r_{\mathrm{sh}}$ and $V_{\mathrm{sh}}$ the radius and velocity of the shock, respectively (Svirski et al. 2012).

At later times, when the mass of the swept-up CSM is comparable to, or larger than, the ejected mass, the system can either enter a phase of energy (the Sedov-Taylor phase) or momentum conservation ("snowplow" phase; Svirski et al. 2012), which, in both cases, would result in a steeper decay of the observed luminosity. If the shock reaches the outer boundary of the dense CSM before this phase occurs, an even steeper drop in luminosity takes place.

Assuming spherical symmetry, Chevalier (1982b) showed that the density profiles of the SN ejecta and dense CSM can be described by power laws of the form $\rho_{\mathrm{ej}} \propto t^{-3}(r / t)^{-n}$ and $\rho_{\mathrm{CSM}}=q r^{-s}$, respectively. In a wind profile $(s=2)$, the density profile of the surrounding material can be expressed by $\rho_{\mathrm{w}}=\dot{M} /\left(4 \pi u_{\mathrm{w}} r^{2}\right)$ (see, e.g., Chevalier \& Fransson 2017), and therefore when we know the normalization constant $q$, it is possible to infer the mass-loss rate of the progenitor star during the last stages of its evolution ${ }^{12}$.

Assuming that the shock breakout occurs at $\tau \approx c / V$, Ofek et al. (2014b) showed that the value of $q$ can be estimated using

$q=\frac{c}{\kappa}(s-1)\left(\frac{n-s}{n-3}\right)^{s-1} V_{\mathrm{bo}}^{s-2} t_{\mathrm{bo}}^{s-1}$,

12 Hereafter we adopt the nomenclature used by Chevalier (1982a), while in Ofek et al. (2014b) $q, n$, and $s$ are called $K, m$, and $w$, respectively. where $\kappa$ is the opacity $\left(\kappa=0.34 \mathrm{~cm}^{2} \mathrm{~g}^{-1}\right.$ for electron scattering in a H-rich gas), $c$ the speed of light, and $V_{\text {bo }}$ is the shock velocity at the shock breakout $V_{s}\left(t_{\mathrm{bo}}\right)$. Therefore, for $s=2, q$ is only a function of $n$ and $t_{\mathrm{bo}}$. Using $L(t)=\epsilon \dot{M} V_{s}(t)^{3} /\left(2 u_{\mathrm{w}}\right)$, $V_{s}\left(t_{\mathrm{bo}}\right)$ can be expressed as a function of the energy conversion efficiency $\epsilon$

$V_{\mathrm{bo}}=t_{\mathrm{bo}}^{(\alpha-1) / 3}\left[2 \pi \epsilon \frac{n-s}{n-3}(s-1) \frac{c}{\kappa L_{0}}\right]^{-1 / 3}$,

with $L_{0}$ directly derived by fitting the bolometric light curve before the break with a power law. In a wind profile, $q=\dot{M} / 4 \pi u_{\mathrm{w}}$ and the mass-loss rate of the progenitor star can be derived directly from the density profile of the CSM as a function of the wind expansion velocity $u_{\mathrm{w}}$, as Eq. (5) loses its dependence on $V_{\text {bo }}$. The mass of the CSM swept up by the shock at the time $t$ can be expressed as a function of $L_{0}, t_{\text {bo }}, n$ and $s$ (see Eq. (21) in Ofek et al. 2014b), which, in a wind profile, can be expressed by:

$M_{\mathrm{CSM}}=4 \pi c\left(\frac{n-2}{n-3}\right)^{5 / 3}\left(\frac{2 \pi c \epsilon}{\kappa^{2} L_{0}}\right)^{-1 / 3} t_{\text {bo }}^{2 / 3} t^{(n-3) /(n-2)}$.

The pseudo-bolometric light curve of SN 2015da can be interpreted as the result of a $\mathrm{SN}$ shock propagating in a dense wind, with photon diffusion in the dense H-rich CSM being the dominant mechanism until $t \simeq+150 \mathrm{~d}$. This epoch corresponds to the onset of the broken power law describing the light curve, when the energy input from the shock directly corresponds to the observed luminosity. The "shock breakout" through the dense wind can then be estimated from the peak of the light curve at $\simeq 30 \mathrm{~d}$ (see Fig. 7), while the observed break at $t \simeq+290 \mathrm{~d}$ corresponds to the onset of the snowplow phase. On the other hand, as shown by Moriya (2014), this would result in a modest decay rate, and a more likely interpretation is the propagation of the shock into a CSM with a lower density, as also inferred from the narrow P-Cygni in the $\mathrm{H} \alpha$ profile (see Sect. 3.2.1).

At $t \geq+150 \mathrm{~d}$, the bolometric light curve of SN 2015da is well-reproduced by a broken power law, with an early luminosity evolution described by $L=9.59 \times 10^{49} t^{-0.92} \mathrm{erg} \mathrm{s}^{-1}$ followed by a much steeper decline with $L \propto t^{-3.98} \mathrm{erg} \mathrm{s}^{-1}$ until $+1233 \mathrm{~d}$ (subtracting the contribution of the IR luminosity at $t \geq+433 \mathrm{~d}$; see Sect. 3.1.1), while the change in the power law index occurs around $+290 \mathrm{~d}$.

Ofek et al. (2014b) showed that the value of $n$ is related to the power law index $\alpha$ through the equation:

$\alpha \equiv \frac{(2-s)(s-3)+3(s-3)}{n-s}$,

which, in a wind profile, gives $n=5.26$ for $\alpha=-0.92$. This value is considerably lower than that inferred for SN 2010jl $(n \simeq$ 7.6; Fransson et al. 2014). The relative time interval between the diffusion phase and break in the light curve is, however, considerably shorter for SN 2015da, which makes the determination of the power law index more uncertain.

Assuming that the time of the shock breakout coincides with the peak in luminosity in the diffusion dominated phase, $t_{\mathrm{bo}} \simeq 30 \mathrm{~d}$, and a wind velocity $u_{\mathrm{w}} \simeq 100 \mathrm{~km} \mathrm{~s}^{-1}$ (see Sect. 3.2.1), Eq. (5) gives a mass-loss rate $\dot{M}=0.66 M_{\odot} \mathrm{yr}^{-1}$ for $n=5.26$. Equation ( 7 ) then gives a total mass of $\simeq 5.48(\epsilon / 0.25)^{-1 / 3} M_{\odot}$ for the CSM swept up by the SN shock at $t=t_{\text {break }}=290 \mathrm{~d}$. Here, we scaled the efficiency to $\epsilon=0.25$ (Ofek et al. 2014a). Some support for a value of $\epsilon$ in this range comes from two-dimensional 
radiation hydrodynamics simulations of Type IIn SNe (Vlasis et al. 2016), who found an efficiency of $\sim 30 \%$ for the conversion of kinetic energy to radiation. We emphasize, however, that this value is uncertain.

Alternatively, after the photon diffusion phase, the bolometric light curve can be expressed (for $s=2$ ) as:

$$
\begin{aligned}
L(t)= & 8.51 \times 10^{42} \times \epsilon\left(\frac{\dot{M}}{0.1 M_{\odot} \mathrm{yr}^{-1}}\right)\left(\frac{u_{\mathrm{w}}}{100 \mathrm{~km} \mathrm{~s}^{-1}}\right)^{-1} \\
& \times\left(\frac{V_{\text {s,break }}}{3000 \mathrm{~km} \mathrm{~s}^{-1}}\right)^{3}\left(\frac{t_{\text {break }}}{320 \mathrm{~d}}\right)^{\alpha} \mathrm{erg} \mathrm{s}^{-1}
\end{aligned}
$$

(Fransson et al. 2014). Equation (9) gives the bolometric luminosity at $t_{\text {break }}$ as a function of the shock velocity at this epoch, $V_{\text {break }}$ (or any other epoch). The mass of the swept up CSM can then be obtained from the same relation used to get Eq. (7):

$M_{\mathrm{CSM}}=\frac{n-3}{n-2} \dot{M} \frac{V_{\mathrm{s}}}{u_{\mathrm{w}}} t_{\text {break }}$.

For low mass-loss rates, $V_{\text {bo }}$ can be inferred from line profiles often observed from the shocked gas of interacting transients (see, e.g., the cases of SNe 1988Z, 2005ip and 2006jd; Turatto et al. 1993; Stritzinger et al. 2012, respectively). However, SN 2015da shows symmetric line profiles with broad wings typical of electron scattering from an outer un-shocked CSM at all epochs (see Sect. 3.2). Therefore, we could not estimate the velocity of the shock at the breakout directly from the spectra. Alternatively, the shock velocity at the time of the breakout can be derived using Eq. (6) for given values of $\epsilon$, resulting in $\simeq 6150(\epsilon / 0.25)^{-1 / 3} \mathrm{~km} \mathrm{~s}^{-1}$ at $t=t_{b 0}=30 \mathrm{~d}$, and $\simeq 1440(\epsilon / 0.25)^{-1 / 3} \mathrm{~km} \mathrm{~s}^{-1}$ at $t=t_{\text {break }}=290 \mathrm{~d}$.

Assuming a typical velocity $V_{\mathrm{s}} \simeq 3000 \mathrm{~km} \mathrm{~s}^{-1}$ at an age of one year, as inferred for other Type IIn SNe such as SNe 2010jl (Fransson et al. 2014) or 2013L (Taddia et al. in prep.), we can infer independent values for $\dot{M}$ and the mass of the swept up CSM, obtaining (adopting $\epsilon=0.25$ ) $\dot{M}=0.62\left(V_{s} / 3000 \mathrm{~km} \mathrm{~s}^{-1}\right)^{-3} M_{\odot} \mathrm{yr}^{-1}$, in agreement with our former estimate. The agreement between these two estimates gives some extra confidence in the choice of this parameter. The total CSM mass from Eq. (10) is therefore $M_{\mathrm{CSM}} \simeq$ $10.34(\epsilon / 0.25)^{-1}\left(V_{s} / 3000 \mathrm{~km} \mathrm{~s}^{-1}\right)^{-2} M_{\odot}$. Again, we point to the considerable uncertainty in this estimate from the assumed values of $V_{\mathrm{s}}$ and $\epsilon$. In addition, we did not take into account possible variations in the kinetic energy conversion efficiency $\epsilon$ due to a high density gradient within the shocked regions (see, e.g., Tsuna et al. 2019).

It is of some interest to compare our simplified modeling with the detailed simulations by Dessart et al. (2015), using the Eulerian radiation-hydrodynamics code HERACLES (González et al. 2007). Although these were designed to model SN 2010j1 and are one-dimensional simulations, they are of interest for understanding the effects of photon diffusion on the observed light curve through a qualitatively visual comparison.

Figure 8 shows that models $\mathrm{X}\left(\dot{M}=0.1 M_{\odot} \mathrm{yr}^{-1}\right)$ and $\mathrm{Xm} 3$ $\left(\dot{M}=0.3 M_{\odot} \mathrm{yr}^{-1}\right)$ are able to roughly reproduce the early evolution of SN 2015da, with comparable rise times, although with lower luminosities. Model Xm6, with a higher mass-loss rate $\left(0.6 M_{\odot} \mathrm{yr}^{-1}\right)$, gives roughly the correct luminosity, but with a slower rise time, while $\mathrm{Xe} 3\left(\dot{M}=0.1 M_{\odot} \mathrm{yr}^{-1}\right.$ and total energy of $3 \times 10^{51} \mathrm{erg}$ ) seems to reproduce the rise time and the early (up to $\simeq 150 \mathrm{~d}$ after explosion) part of the light curve after maximum well, although with a much higher peak luminosity. However, we note that the early light curve is uncertain due to the large

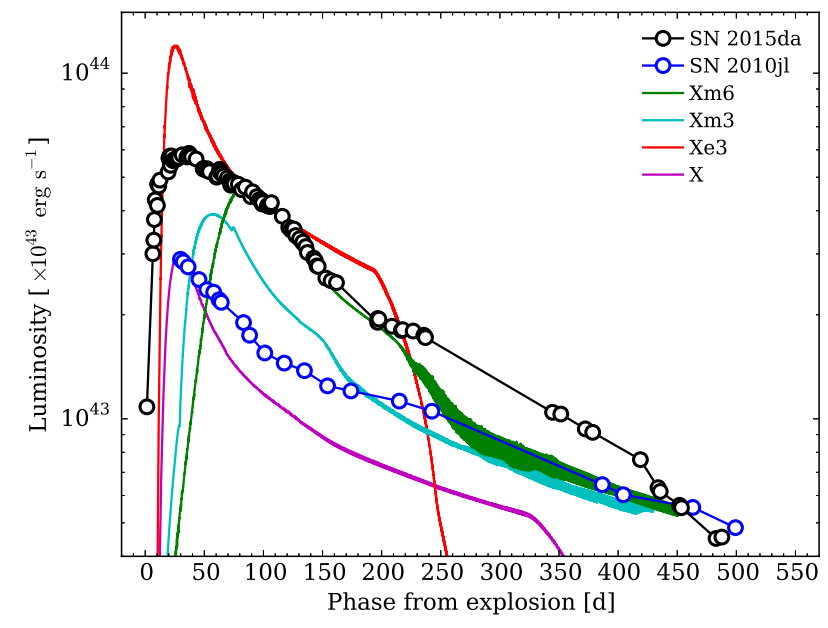

Fig. 8. Bolometric light curve of SN 2015da compared to models presented in Dessart et al. (2015) and bolometric light curve of SN 2010j1 (computed as in Fransson et al. 2014). Model X has $\dot{M}=0.1 M_{\odot} \mathrm{yr}^{-1}$, model Xm3 $\dot{M}=0.3 M_{\odot} \mathrm{yr}^{-1}$, and model Xm6 $\dot{M}=0.6 M_{\odot} \mathrm{yr}^{-1}$. In all cases, the total energy is $10^{51} \mathrm{erg}$. Model Xe3 has $\dot{M}=0.1 M_{\odot} \mathrm{yr}^{-1}$ and total energy $3 \times 10^{51} \mathrm{erg}$.

bolometric correction from the assumed spectral shape in the UV. All these models correspond to a $9.8 M_{\odot}$ inner ejecta breaking through a dense CSM (with a total mass of $2.89 M_{\odot}$ ) with a wind density profile, $u_{\mathrm{w}}=100 \mathrm{~km} \mathrm{~s}^{-1}$, a constant temperature of $2000 \mathrm{~K}$, and total kinetic energy of $10^{51} \mathrm{erg}$. While a more detailed modeling tuned to the observations of SN 2015da would be of obvious interest, a rough comparison between radiative transfer models and our estimates provides a comparable mass loss, although with a higher total CSM mass.

To account for these high values, we have to assume that a large fraction of the surrounding CSM was expelled by the progenitor star through repeated massive eruptive events like those occasionally experienced by LBV stars (see the case of the Great Eruption of $\eta$ Car, e.g., Morris et al. 1999; Smith et al. 2003). On the other hand, the mass-loss rate and CSM mass inferred through Eqs. (5) and (7) are strongly dependent on the assumed value of $t_{\mathrm{bo}}$ and $s$. Ofek et al. (2014a) showed that for $s<2$, the shock is expected to break through near the edge of the CSM, and the model would not give a light curve with a power law decay lasting long enough to reproduce the light curve of SN 2010j1. We note, however, that SN 2015da shows a somewhat different behavior at $t \leq t_{\text {break }}$, with a significantly shorter first decline than that observed in SN 2010j1 (see Fig. 7).

\subsection{Spectroscopy}

The optical spectral evolution of SN 2015da is shown in Fig. 9, which includes a selection of spectra with the highest signalto-noise ratios (S/Ns) and resolutions. The entire set of spectroscopic data is available, along with the photometric tables, through the Weizmann Interactive Supernova data REPository (WISEREP ${ }^{13}$; Yaron \& Gal-Yam 2012). Our 2 NIR spectra are shown in Fig. 10, while a complete log of the spectroscopic observations is reported in Table A.6 and shown in Fig. A.1 in Appendix A.2.

At early phases, the spectra show classical features of Type IIn SNe, such as a blue continuum with prominent narrow $\mathrm{H}$ I $(\mathrm{H} \alpha$ to $\mathrm{H} \epsilon)$; see Fig. 11 showing the early evolution of $\mathrm{H} \alpha$ and $\mathrm{He} \mathrm{I}$ ( $\lambda 5875$ and $\lambda 7065)$ lines in emission. The strong

13 https://wiserep.weizmann.ac.il/ 

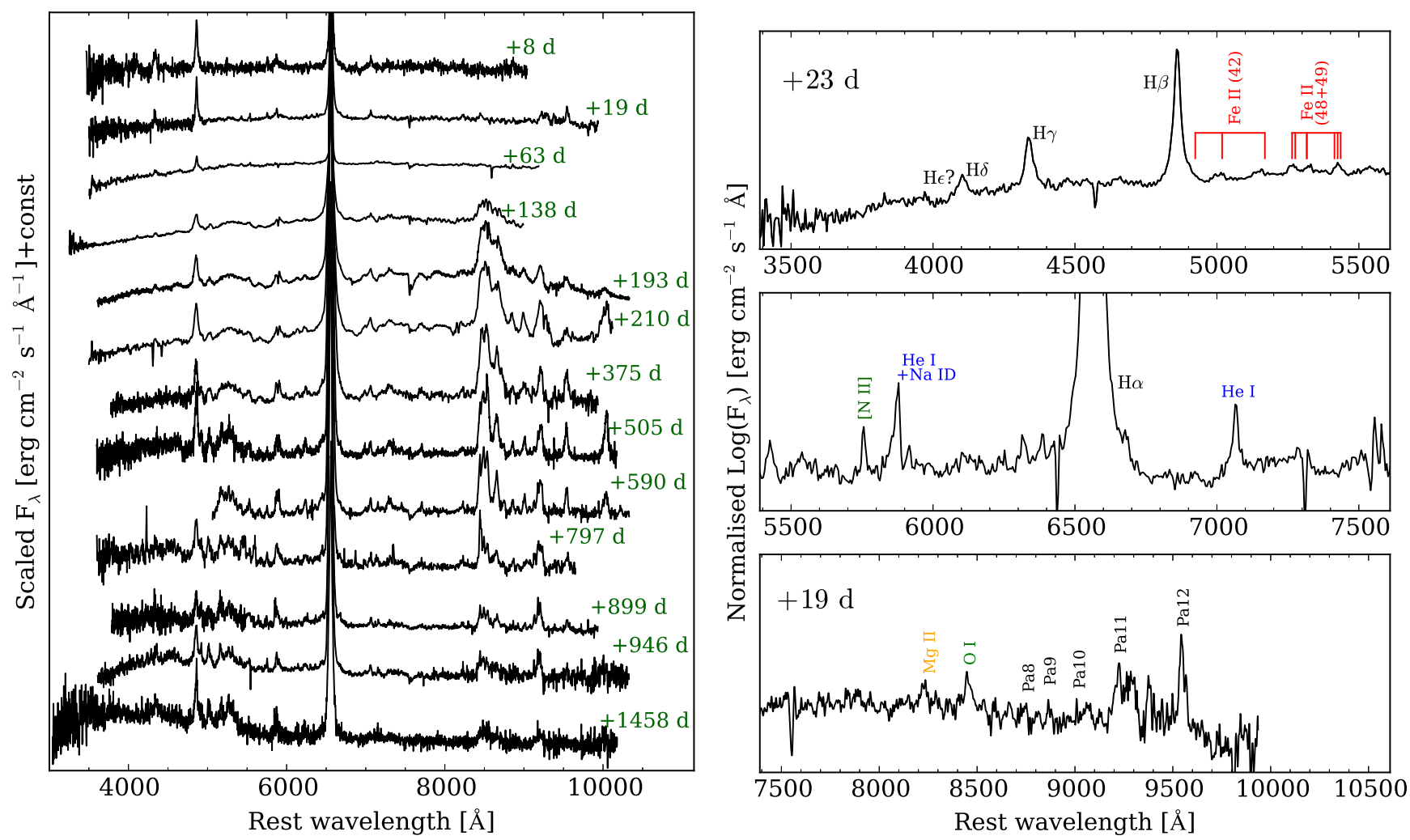

Fig. 9. Left: selection of optical spectra of SN 2015da with highest S/N, spectral coverage and resolution. Spectra were not corrected for extinction to facilitate the comparison at wavelengths bluer than $\simeq 5000 \AA$. Right: line identification on the $+23 \mathrm{~d}$ (first and second panel) and $+19 \mathrm{~d}($ bottom panel) spectra. Phases refer to the estimated epoch of the explosion.
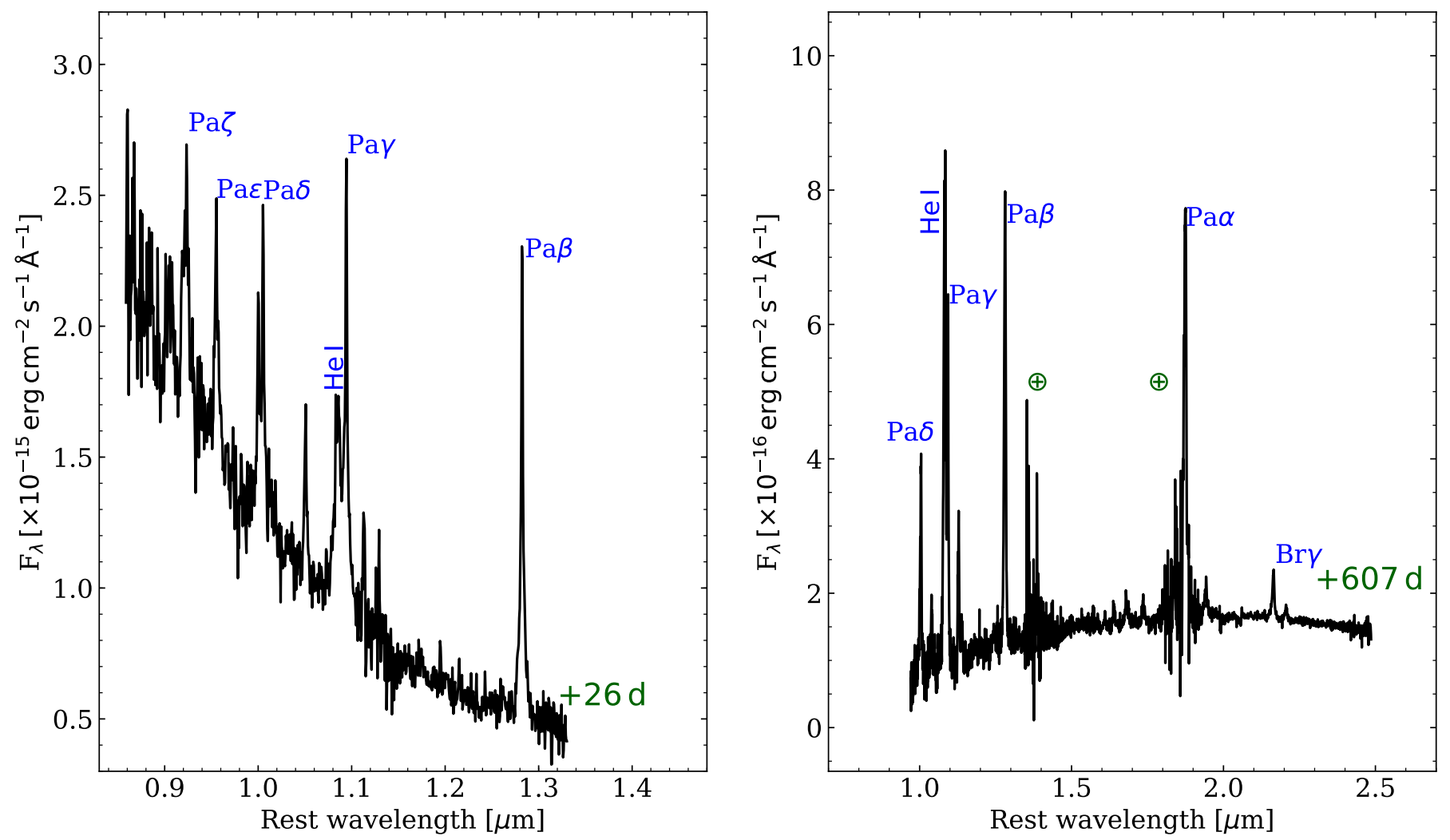

Fig. 10. NIR spectra of SN $2015 \mathrm{da}$ at $+26 \mathrm{~d}($ left $)$ and $+607 \mathrm{~d}($ right $)$. Phases refer to the estimated epoch of the explosion. 
Na I D doublet $(\lambda \lambda 5890,5896)$ is clearly visible until $+193 d$, suggesting a highly extinguished local environment (see Sect. 2). At later phases, the spectral continuum fades and the He I $\lambda 5875$ line dominates the spectral flux at these wavelengths. From the positions of the minima of the $\mathrm{Na}$ I D features observed in the DEIMOS spectrum we inferred a heliocentric recessional velocity of $\simeq 2000 \mathrm{~km} \mathrm{~s}^{-1}$ that we adopt to set the observed spectra at rest wavelengths.

Early spectra $(t \simeq+8 \mathrm{~d})$ also show narrow circumstellar [N II] $\lambda 5755$, broad O I $\lambda 8446$ and narrow NIR H I features ( $\mathrm{Pa} 8$ to $\mathrm{Pa}$ 12), while Fe II lines (multiplets 42, 48 and 49) are visible, although faint, already at $+23 \mathrm{~d}$, becoming more evident at later phases $(t \geq+110 \mathrm{~d})$, where they start to contribute significantly to the shape of the blue pseudo-continuum (at $\lambda \lesssim 5800 \AA$ ). At $+19 \mathrm{~d}$ we also identify $\mathrm{Mg}$ II $\lambda \lambda 7877,7896, \lambda \lambda 8214,8235$, and $\lambda \lambda 9218,9244$. The NIR Ca II triplet starts to dominate the red part of the optical spectra from $t \simeq+138 \mathrm{~d}$, becoming most prominent at $\simeq 505 \mathrm{~d}$, when it starts to fade with respect to the spectral continuum.

The NIR H I lines become progressively stronger up to $+590 \mathrm{~d}$ whereafter they slowly decrease in strength. At $+35 \mathrm{~d}$, the NIR spectrum is dominated by narrow $\mathrm{HI}(\mathrm{Pa} \beta$ to $\mathrm{Pa} \zeta)$ and He I ( $\lambda 10830)$ lines.

Fitting a BB to the spectral continuum, we inferred the temperature evolution of the pseudo photosphere, slowly declining from $\simeq 13430 \pm 355 \mathrm{~K}$ to $\simeq 7180 \pm 510 \mathrm{~K}$ during the first $\simeq 240 \mathrm{~d}$ after explosion, in agreement with the one inferred from the SEDs (see Sect. 3.1.1). From $t \simeq 193 \mathrm{~d}$, the spectra show a blue excess at wavelengths shorter than $\simeq 5500 \AA$, becoming more evident at later epochs, observed throughout the remaining $\sim 1300 \mathrm{~d}$ of the spectroscopic monitoring. The source of the excess is likely not thermal, since spectra at these wavelengths $(\lambda<5500-5800 \AA)$ are significantly affected by strong Fe II blends. On the other hand, late $(t \gtrsim 100 \mathrm{~d})$ blue excesses are common in interacting transients (see the cases of SNe 2006jc; Foley et al. 2007; Pastorello et al. 2007 and 2005ip; Smith et al. $2009 \mathrm{~b}$ ) and are likely due to the contribution of fluorescence from a number of blended Fe lines or to a "revitalized" late-time ejecta-CSM interaction with a low energy conversion efficiency (see, e.g., Smith et al. 2009b).

\subsubsection{The DEIMOS spectrum}

A moderate-resolution spectrum $(R \simeq 3600$ from the $[\mathrm{OI}]$ sky lines) was obtained on 2015 February 23.66 UT (JD = 2457 077.16, corresponding to $t=+46 \mathrm{~d}$; Program ID U063D, PI Filippenko), with the DEep Imaging Multi-Object Spectrograph (DEIMOS; Faber et al. 2003) mounted at the $10 \mathrm{~m}$ Keck II telescope at Mauna Kea. These data are available in the public section of the Keck Observatory Archive $\left(\mathrm{KOA}^{14}\right)$.

The spectrum shows many marginally resolved Fe II lines with P-Cygni absorption features (multiplets 40, 42, 46, 48, 49, 74 ) or purely in emission (200, 40 apart from $\lambda 6516$ and 49, with the possible exception of $\lambda 6113$ ). We also identified a number of narrow lines purely in emission corresponding to other transitions, namely [N II] $\lambda 5755, \mathrm{He}$ I $\lambda 5875$ and Si II $\lambda \lambda 6347,6371$ as well as a few other unidentified lines at 5568, 5587, 6318, 6332 , and 6384/6385 $\AA$. The Fe II $\lambda 5169$ line, typically used to infer the photospheric expansion velocity in SNe, shows a faint narrow emission with a structured absorption component, a minimum of $\simeq 10 \mathrm{~km} \mathrm{~s}^{-1}$, and a blue wing, possibly contaminated by a second component, extending up to $\simeq 110 \mathrm{~km} \mathrm{~s}^{-1}$. Fe II lines are

\footnotetext{
14 https://www2 . keck .hawaii . edu/koa/public/koa.php
}

blueshifted ${ }^{15}$ by $\simeq 40-50 \mathrm{~km} \mathrm{~s}^{-1}$, from which we infer minima of $\simeq 50 \mathrm{~km} \mathrm{~s}^{-1}$ with a terminal velocity of $100-110 \mathrm{~km} \mathrm{~s}^{-1}$.

In Fig. 12, we zoom in on the DEIMOS spectrum in the region of $\mathrm{H} \alpha$ showing a narrow $\mathrm{P}$ Cygni feature with a minimum blueshifted by $\simeq 55 \mathrm{~km} \mathrm{~s}^{-1}$, and a terminal velocity of $\simeq 110 \mathrm{~km} \mathrm{~s}^{-1}$ on top of a broader profile, typical of electron scattering, with wings showing a full-width-at-zero-intensity (FWZI) of $\simeq 3 \times 10^{3} \mathrm{~km} \mathrm{~s}^{-1}$.

To illustrate the dominance of electron scattering in the formation of the line profiles, we used the same Monte Carlo code as in Fransson et al. (2014). The input photons from recombination and collisions are emitted from the ionized region of the slowly moving "precursor shock" (Sutherland \& Dopita 2017) and then undergo electron scattering in the same region, although most of them are emitted close to the shock. The main parameters of the fit are the optical depth to electron scattering, $\tau_{\mathrm{e}}$ and the electron temperature, $T_{\mathrm{e}}$. Since the change in frequency in each scattering is $\propto T_{\mathrm{e}}^{1 / 2}$, to obtain a given FWHM, we need $\tau_{\mathrm{e}} \propto T_{\mathrm{e}}^{-1 / 2}$. The two parameters are therefore degenerate. In our calculations, we assumed $T_{\mathrm{e}}=10^{4} \mathrm{~K}$, and we did not attempt to model the resonance scattering by the $\mathrm{H} \alpha$ line giving rise to the narrow P Cygni profile.

In Fig. 12, we show the resulting fit, where the broad wings are well-reproduced by an exponential profile typical of electron scattering (Huang \& Chevalier 2018). To obtain the observed FWHM at this epoch, we need $\tau_{\mathrm{e}} \approx 6.0\left(T_{\mathrm{e}} / 10^{4} \mathrm{~K}\right)^{-1 / 2}$. This value is similar to the one obtained for SN 2010jl (Fransson et al. 2014), and it shows that the gas is optically thick to electron scattering. The narrow line in the model is due to unscattered photons at zero velocity. These photons are scattered by the $\mathrm{H} \alpha$ line itself and form part of the P Cygni profile below $\sim 200 \mathrm{~km} \mathrm{~s}^{-1}$. The fact that this results in a P Cygni profile means that the emission must come from a more extended region, producing both the absorption and emission component.

An immediate conclusion is that the emission from the inner parts of the ejecta (with respect to the forward shock), have an even higher optical depth. Emission lines from this region would therefore be washed out into a continuum, explaining why we do not observe broad lines from the expanding ejecta or post-shock gas.

\subsubsection{Evolution of the $\mathrm{H}$ lines}

Physical quantities inferred from the main Balmer lines (i.e., $\mathrm{H} \alpha$ and $\mathrm{H} \beta$ ) and described in this section are reported in Table 5, as obtained through the IRAF task SPLOT, including FWHM velocities and equivalent widths (EWs). EWs for $\mathrm{H} \alpha$ and $\mathrm{H} \beta$ show average values of $\sim 880 \AA$ and $\sim 75 \AA$, respectively, in agreement with the distribution of values inferred for the sample of SNe IIn presented in Silverman et al. (2013), who also proposed weaker $\mathrm{H} \beta$ lines (with $\mathrm{EW} \simeq 6 \AA$, as well as the absence of He I $\lambda 5876$ features) as a hallmark feature of Ia-CSM SNe. The values inferred for SN $2015 \mathrm{da}$ argue against a Type Ia-CSM interpretation for this object.

At $t<+63 \mathrm{~d}$, Balmer lines show narrow profiles purely in emission, with roughly constant FWHM velocities of $\simeq 10^{3} \mathrm{~km} \mathrm{~s}^{-1}$ and $\simeq 1.7 \times 10^{3} \mathrm{~km} \mathrm{~s}^{-1}$ for $\mathrm{H} \alpha$ and $\mathrm{H} \beta$, respectively. All Balmer lines are well reproduced using a Lorentzian profile, and we do not see any trace of the narrow component observed in the higher resolution DEIMOS spectrum obtained at $+46 \mathrm{~d}$ (see Sect. 3.2.1). This is most likely due to an effect of resolution,

15 With the exception of multiplet 74 , which shows peaks almost at rest wavelengths. 

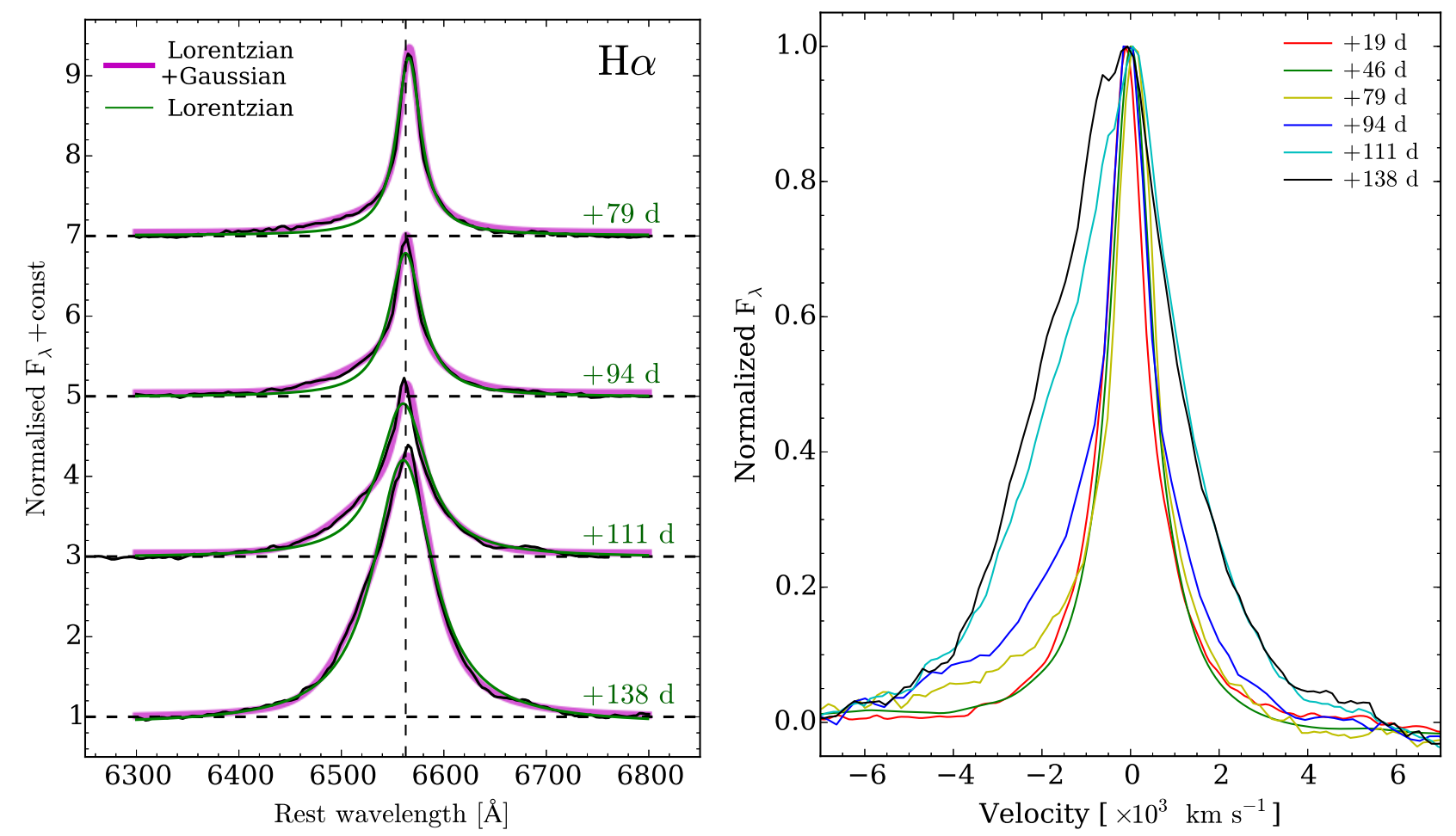

Fig. 11. Left: fit to the $\mathrm{H} \alpha$ line profiles on the $+79 \mathrm{~d},+94 \mathrm{~d},+111 \mathrm{~d}$ and $+138 \mathrm{~d}$ spectra using a combination of Lorentzian and Gaussian functions. Right: evolution of the continuum-subtracted $\mathrm{H} \alpha$ profile over a selection of phases. The line profiles were normalized to their peak values in order to highlight their evolution. Phases refer to the estimated epoch of the explosion.

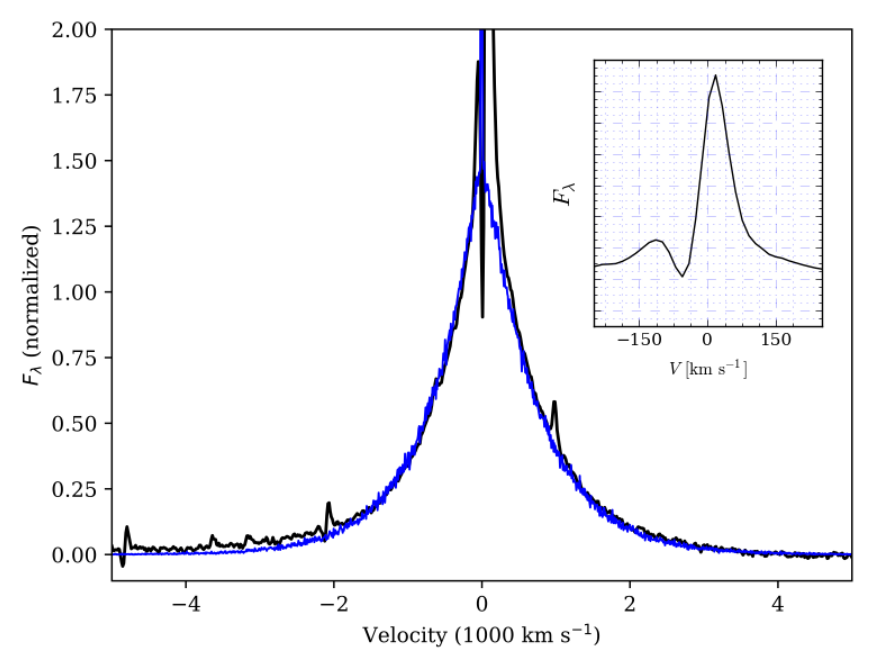

Fig. 12. $\mathrm{H} \alpha$ line profile observed in the $+46 \mathrm{~d}$ Keck DEIMOS spectrum of SN 2015da along with a fit to the line profile from electron scattering. The line profile below $\pm 200 \mathrm{~km} \mathrm{~s}^{-1}$, in the model from un-scattered photons, is affected by the resonance line scattering in the $\mathrm{H} \alpha$ transition and was not modeled.

as suggested by the appearance of the narrow component in the moderate-resolution spectra obtained at $+505 \leq t \leq+606 \mathrm{~d}$, all having resolutions $\lesssim 10 \AA$ in the $6300-6800 \AA$ region.

From $+79 \mathrm{~d}$, we note small deviations from a single Lorentzian profile in the blue wing of $\mathrm{H} \alpha$ (see Fig. 11) and a second Gaussian component is required to fit the entire profile. The $\mathrm{H} \beta$ line profile, on the other hand, is well-reproduced by a single Lorentzian component at all phases. A second component is required also at later phases, although at $+138 \mathrm{~d} \mathrm{H} \alpha$ does not show significant asymmetries, and a single Lorentzian component is again sufficient to fit the entire profile (see Fig. 11). In the early NIR spectrum $(+26 d)$, Paschen lines are marginally resolved $\left(F W H M \simeq 600 \mathrm{~km} \mathrm{~s}^{-1}\right)$ and show symmetric profiles centered at the corresponding rest wavelengths. At later phases $(+607 \mathrm{~d})$, we notice a broadening $(F W H M \simeq$ $1100-1300 \mathrm{~km} \mathrm{~s}^{-1}$ ) in the $\mathrm{Pa} \gamma$ and $\mathrm{Pa} \beta$ lines, with slightly blueshifted peaks of $\simeq 100 \mathrm{~km} \mathrm{~s}^{-1}\left(\simeq 40 \mathrm{~km} \mathrm{~s}^{-1}\right.$ for Pa $\gamma$, which is strongly contaminated by the prominent He I $\lambda 10830$ line), although the overall profiles are still well-reproduced by single symmetric Lorentzian (or Gaussian, for marginally resolved lines) profiles.

We note a similar evolution in $\mathrm{H} \alpha$, with the centroid of the line progressively shifting toward bluer wavelengths with time, up to $300-500 \mathrm{~km} \mathrm{~s}^{-1}$ at $t \gtrsim 833 \mathrm{~d}$, where the uncertainty is due to the different $\mathrm{S} / \mathrm{N}$ and resolutions of the spectra. This is highlighted in Fig. 11 (right panel), showing the evolution of $\mathrm{H} \alpha$ over selected phases. The $\mathrm{H} \alpha$ profile shows a broadening at $t \gtrsim 100 \mathrm{~d}$, which might be due to a gradual emergence of the emission from the shock, while the overall profile remains symmetric (see below).

In SN 2010jl, wavelength-dependent asymmetries and the apparent dimming of the red wings of emission components was used by Smith et al. (2012) and Gall et al. (2014) to suggest rapid dust formation in the $\mathrm{SN}$ ejecta as the possible cause of the IR excess. However, Fransson et al. (2014) showed that the profile of $\mathrm{H} \alpha$ remains symmetric with respect to a shifted centroid and attributed this shift to a bulk velocity of the emitting shell or to acceleration of the un-shocked CSM by the radiation field generated in the inner shocked regions. Following Fransson et al. (2014), we therefore mirrored the red wing of $\mathrm{H} \alpha$ with respect to the computed centroid of the line profile at each epoch. The resulting profiles are shown in Fig. 13 for a selection of spectra 
Table 5. Main parameters of the BB fit to the observed SED evolution of SN 2015da.

\begin{tabular}{|c|c|c|c|c|}
\hline JD & $\begin{array}{l}\text { Phase } \\
\text { (d) }\end{array}$ & $\begin{array}{c}\text { Luminosity }(\mathrm{H} \alpha / \mathrm{H} \beta) \\
\left(\mathrm{erg} \mathrm{s}^{-1}\right)\end{array}$ & $\begin{array}{c}\mathrm{EW}(\mathrm{H} \alpha / \mathrm{H} \beta) \\
(\AA)\end{array}$ & $\begin{array}{c}F W H M(\mathrm{H} \alpha / \mathrm{H} \beta) \\
\left(\mathrm{km} \mathrm{s}^{-1}\right)\end{array}$ \\
\hline 2457039.37 & +8 & $5.78 / 3.62$ & $370 / 110$ & $1425 / 1970$ \\
\hline 2457041.37 & +10 & $6.45 / 3.43$ & $385 / 80$ & $1630 / 2400$ \\
\hline 2457042.38 & +11 & $6.11 / 3.43$ & $320 / 85$ & $1575 / 2025$ \\
\hline 2457049.99 & +19 & $7.55 / 4.15$ & $240 / 85$ & $1350 / 1525$ \\
\hline 2457050.37 & +19 & $6.55 / 4.06$ & $240 / 85$ & $1410 / 2100$ \\
\hline 2457053.67 & +23 & $7.00 / 3.93$ & $275 / 75$ & $1335 / 2060$ \\
\hline 2457057.34 & +26 & $6.60 / 2.53$ & $230 / 65$ & $1415 / 1960$ \\
\hline 2457063.55 & +33 & $5.60 / 3.27$ & $175 / 55$ & $1275 / 2370$ \\
\hline 2457067.35 & +36 & $5.45 / 2.94$ & $165 / 50$ & $1440 / 2235$ \\
\hline 2457077.16 & +46 & $3.95 / 1.27$ & $105 / 20$ & $540 / 1060^{(a)}$ \\
\hline 2457093.52 & +63 & $3.05 / 1.32$ & $90 / 20$ & $1135 / 1930$ \\
\hline 2457109.59 & +79 & $3.80 / 0.92$ & $90 / 15$ & $1450 / 1980$ \\
\hline 2457119.83 & +89 & $3.15 / 1.20$ & $65 / 20$ & $1590 / 2295$ \\
\hline 2457124.48 & +94 & $3.80 / 0.94$ & $85 / 15$ & $2110 / 2230$ \\
\hline 2457141.62 & +111 & $5.20 / 1.58$ & $1450 / 25$ & $3120 / 3045$ \\
\hline 2457168.64 & +138 & $8.75 / 2.89$ & $265 / 50$ & $3770 / 3230$ \\
\hline 2457185.64 & +155 & $10.85 / 3.15$ & $370 / 65$ & $3710 / 3165$ \\
\hline 2457224.39 & +193 & $11.05 / 3.00$ & $505 / 80$ & $3480 / 3020$ \\
\hline 2457224.43 & +193 & $12.80 / 2.40$ & $655 / 85$ & $3820 / 2530$ \\
\hline 2457241.32 & +210 & $13.75 / 3.10$ & $715 / 105$ & $3700 / 2890$ \\
\hline 2457259.39 & +228 & $13.15 / 3.10$ & $825 / 80$ & $3560 / 3010$ \\
\hline 2457273.30 & +242 & $13.85 / 2.95$ & $880 / 105$ & $3700 / 3030$ \\
\hline 2457359.68 & +329 & $12.00 / 2.10$ & $1250 / 115$ & $3470 / 2560$ \\
\hline 2457406.02 & +375 & $12.35 / 2.00$ & $1040 / 110$ & $3200 / 2230$ \\
\hline 2457465.60 & +435 & $3.60 / 0.50$ & $1940 / 145$ & $2990 / 2350$ \\
\hline 2457535.90 & +505 & $6.60 / 1.20$ & $1190 / 85$ & $2600 / 2300$ \\
\hline 2457562.44 & +531 & $1.35 / 0.30$ & $1095 / 160$ & $2430 / 3060$ \\
\hline 2457603.68 & +573 & $6.10 / 0.90$ & $1550 / 100$ & $2370 / 2130$ \\
\hline 2457621.38 & +590 & $6.00 /-^{(a)}$ & $1735 /-^{(a)}$ & $2335 /-^{(a)}$ \\
\hline 2457626.64 & +596 & $4.25 / 0.70$ & $1235 / 80$ & $2285 / 2180$ \\
\hline 2457636.66 & +606 & $7.70 / 0.45$ & $2265 / 110$ & $2295 / 2430$ \\
\hline 2457644.36 & +613 & $3.20 / 0.35$ & $1150 / 65$ & $2205 / 2780$ \\
\hline 2457728.75 & +698 & $2.25 / 0.40$ & $945 / 75$ & $2080 / 2625$ \\
\hline 2457736.74 & +706 & $1.95 / 0.30$ & $1190 / 60$ & $2130 / 2940$ \\
\hline 2457780.98 & +750 & $2.70 / 0.35$ & $1135 / 80$ & $2110 / 2150$ \\
\hline 2457806.73 & +776 & $1.85 / 0.40$ & $1145 / 75$ & $2030 / 2715$ \\
\hline 2457827.57 & +797 & $1.90 / 0.35$ & $1160 / 65$ & $1995 / 2950$ \\
\hline 2457841.40 & +810 & $1.80 / 0.35$ & $1160 / 75$ & $2010 / 2365$ \\
\hline 2457863.55 & +833 & $2.10 / 0.40$ & $1490 / 110$ & $2040 / 2315$ \\
\hline 2457867.59 & +837 & $1.60 / 0.30$ & $1070 / 80$ & $2000 / 2445$ \\
\hline 2457875.54 & +845 & $1.65 / 0.30$ & $945 / 70$ & $2010 / 2940$ \\
\hline 2457899.58 & +869 & $1.90 / 0.30$ & $1125 / 85$ & $1965 / 2765$ \\
\hline 2457914.52 & +884 & $1.30 / 0.25$ & $940 / 50$ & $2030 / 2620$ \\
\hline 2457929.81 & +899 & $2.65 / 0.25$ & $1360 / 60$ & $2070 / 2250$ \\
\hline 2457951.44 & +920 & $2.00 / 0.35$ & $1155 / 60$ & $2030 / 2235$ \\
\hline 2457961.69 & +931 & $2.10 / 0.40$ & $1285 / 50$ & $2000 / 2605$ \\
\hline 2457968.40 & +937 & $1.50 / 0.25$ & $1300 / 65$ & $2085 / 3135$ \\
\hline 2457977.41 & +946 & $1.35 / 0.30$ & $1030 / 50$ & $2020 / 2330$ \\
\hline 2458121.74 & +1091 & $1.75 / /^{(a)}$ & $1025 /$ - $^{(a)}$ & $2140 /{ }_{-(a)}$ \\
\hline 2458489.12 & +1458 & $1.45 / 0.50$ & $1755 / 95$ & $2340 / 2255$ \\
\hline
\end{tabular}

Notes. ${ }^{(a)}$ Uncertain or missing values due to the limited spectral coverage of the spectrum.

with high S/N and good resolution. As in SN 2010j1, no sign of asymmetries is seen at any epoch, suggesting that a macroscopic velocity is the most likely reason for the blueshift of the $\mathrm{H} \alpha$ profile of SN 2015da.

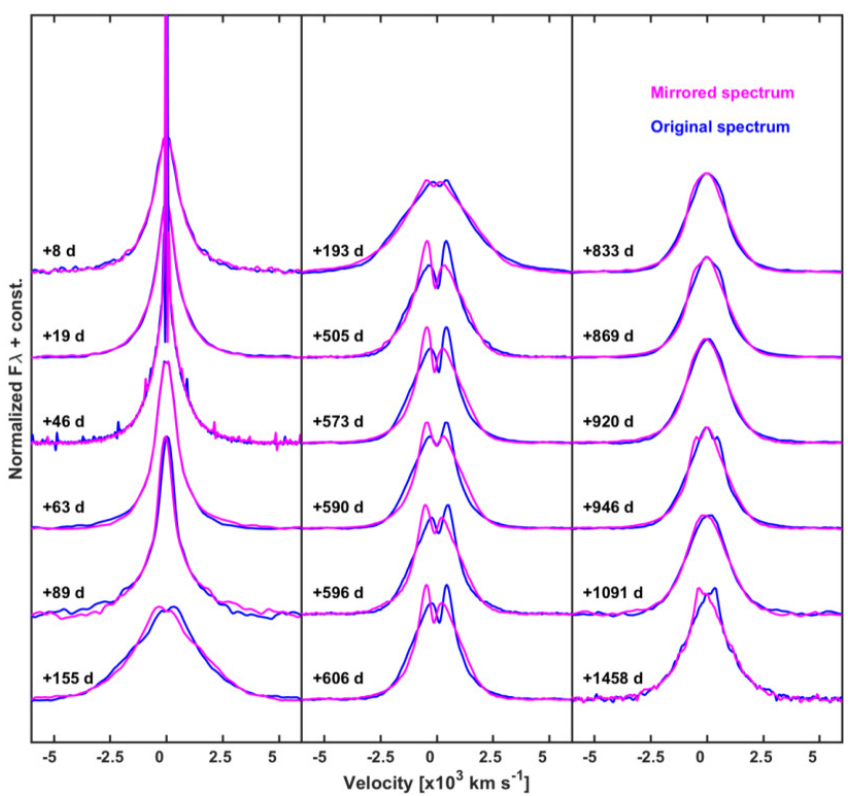

Fig. 13. $\mathrm{H} \alpha$ profiles at selected epochs, redshifted to the line rest wavelength (blue) and mirrored with respect to the computed centroids (magenta). Lines show highly symmetric profiles, with blue and red wings overlapping almost perfectly at all phases. Small asymmetries in the top part of the lines are due to the presence of the narrow P Cygni features, more evident in the higher resolution spectra. Phases refer to the estimated epoch of the explosion.

The evolution of the $\mathrm{H} \alpha$ and $\mathrm{H} \beta$ integrated luminosity is shown in Fig. 14. We note a rapid decline for both lines during the first $63 \mathrm{~d}$, with the luminosity evolving from $\simeq 6.58 / 3.60 \times 10^{41} \mathrm{erg} \mathrm{s}^{-1}$ to $5.46 / 2.94 \times 10^{41} \mathrm{erg} \mathrm{s}^{-1}$ for $\mathrm{H} \alpha / \mathrm{H} \beta$, respectively. The luminosity shows a subsequent rebrightening up to $1.39 / 0.29 \times 10^{42} \mathrm{erg} \mathrm{s}^{-1}$ during the following $\simeq 180 \mathrm{~d}$, with an offset of $\simeq+30 \mathrm{~d}$ with respect to the onset of the re-brightening observed in SN 2010jl (assuming JD = 2455479 as the explosion epoch for SN 2010jl; Fransson et al. 2014). The integrated luminosities show a further decline at later phases, until $+706 \mathrm{~d}$, when it sets at $1.94 / 0.33 \times 10^{41}$, staying roughly constant for the remaining $\simeq 750 \mathrm{~d}$. In SN 2015da, the $\mathrm{H} \alpha / \mathrm{H} \beta$ ratio increases monotonically up to $\simeq+560 \mathrm{~d}$, when it settles at a roughly constant value of $\simeq 5.7$, while at $+1458 \mathrm{~d}$, it drops again to 2.92 , showing a quite different evolution with respect to that of SN 2010jl. This different behavior might be attributed to the blue pseudo continuum contamination of the spectra at $t \gtrsim 228 \mathrm{~d}$, which can bias the integrated luminosity inferred for $\mathrm{H} \beta$ (see Sect. 3.2 and Fig. 14, upper panel).

\subsection{The progenitor star}

The field of SN 2015da was monitored by the Palomar Transient Factory $\left(\mathrm{PTF}^{16}\right)$, which imaged its host galaxy with a roughly constant cadence from 2009 March 17 to 2014 May 28 (see Table A.2). Frames were recovered through the NASA/IPAC Infrared Science Archive ${ }^{17}$. We could not detect traces of preSN variability down to $g \simeq-16.1 \mathrm{mag}$ or $-15 \mathrm{mag}$, assuming a distance of 53.2 Mpc and the total extinction reported in Sect. 2. LBVs are among the most luminous stars known, and typically have absolute magnitudes $M_{\mathrm{R}} \simeq-9$ mag in quiescence. On

\footnotetext{
16 https://www.ptf.caltech.edu/

17 https://irsa.ipac.caltech.edu/
} 


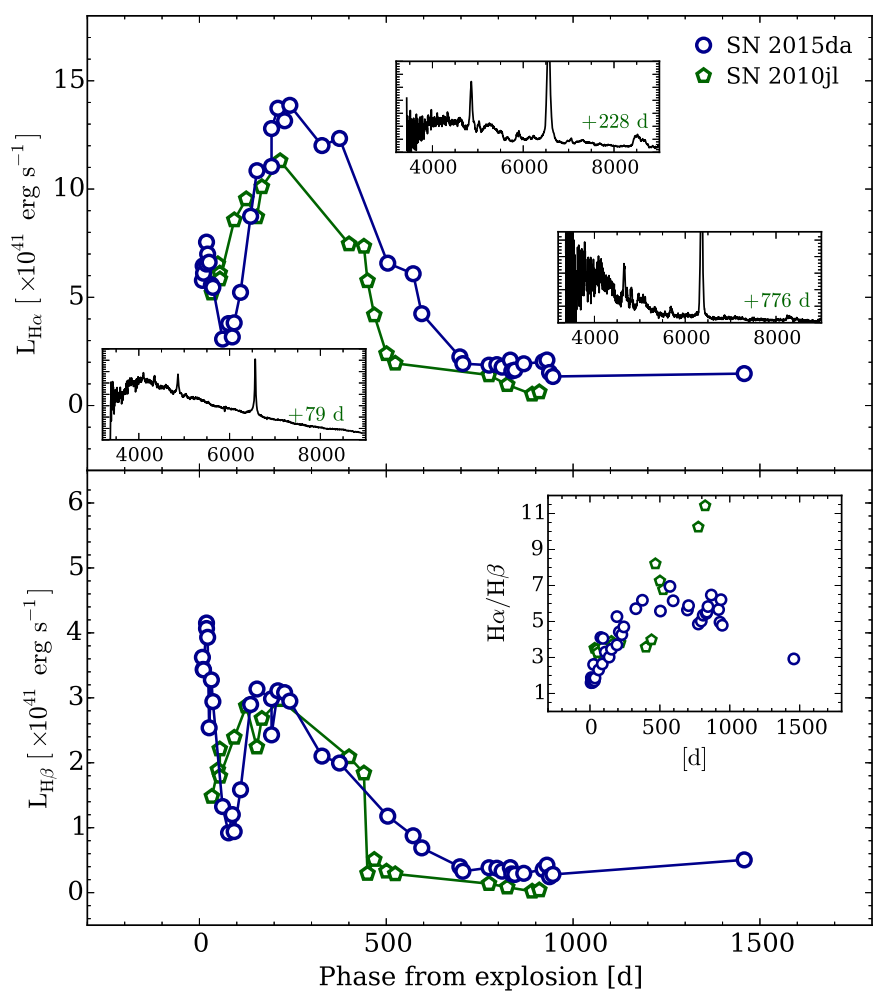

Fig. 14. Evolution of the integrated luminosity of the most prominent Balmer lines $(\mathrm{H} \alpha$ and $\mathrm{H} \beta)$ compared to those inferred for SN 2010j1. Insets in the upper panel show representative spectra during the different phases of the evolution of SN 2015da. The inset in the bottom panel shows the evolution of the $\mathrm{H} \alpha / \mathrm{H} \beta$ ratio compared to that computed for SN 2010j1.

the other hand, they occasionally experience nonterminal major eruptive events, like the ones observed in $\eta$-Car in the 19th century (e.g., Smith \& Frew 2011), producing optical transients that mimic the behavior of SNe IIn (hence dubbed "SN impostors"; Van Dyk et al. 2000; Maund et al. 2006), although with fainter absolute magnitudes $\left(M_{\text {peak }} \simeq-14 \mathrm{mag}\right.$, e.g., Tartaglia et al. 2015, 2016). In addition, we cannot rule out a larger value of reddening in the environment of the progenitor star before explosion. An SN explosion in a dusty environment is expected to produce a dust-free cavity within a radius directly dependent on the peak luminosity of the transient. Dwek (1983) showed that an SN with $L_{\text {peak }}=10^{10} L_{\odot}$ can produce a cavity of $5.1 \times 10^{17} \mathrm{~cm}$, depending on the chemical composition, size of the dust gains, density and optical depth of the shell. Therefore, a larger amount of dust could survive less luminous outbursts. It is therefore possible that the local extinction in the environment of SN 2015da was higher during the pre-SN stages than was estimated in Sect. 2, possibly masking multiple LBV-like outbursts. In this scenario, the available observations would not put sensible constraints on the pre-explosion variability of the precursor.

The prominent narrow [N II] line visible in the spectra almost at all phases is indicative of a possible nitrogen enrichment of the narrow-line region of the CSM, which in turn might indicate a large enrichment of CNO processed gas. Significant CNO enrichments have been observed in SN ejecta or CSM for a small number of CC SNe (see, e.g., Fransson et al. 2005, and references therein), as well as evolved massive stars such as LBVs. Unfortunately, most of the diagnostic lines commonly used for
CNO analyses are out of the observed spectral range (e.g., C III, C IV, N III], N IV], and O III], all at $\lambda<2000 \AA$; Fransson et al. 2014), and we therefore did not have the sufficient spectroscopic coverage or resolution to perform a detailed analysis.

On the other hand, from the narrow $\mathrm{H} \alpha$ P Cygni absorption observed in the higher resolution DEIMOS spectrum at $+46 \mathrm{~d}$, we inferred a wind velocity of $\simeq 100 \mathrm{~km} \mathrm{~s}^{-1}$ ( similar to that of SN 2010jl), suggesting a very massive star as a viable progenitor for SN 2015da. This corresponds to the lower end of the range of typical velocities inferred for LBVs $\left(10^{2}-10^{3}\right.$; e.g. Smith et al. 2011), which, on the other hand, is significantly higher than those displayed by red supergiants (RSG, $10-50 \mathrm{~km} \mathrm{~s}^{-1}$; e.g. Jura \& Kleinmann 1990). In addition, the total mass of the CSM inferred from our analysis of the pseudo-bolometric light curve $\left(\gtrsim 5.5 M_{\odot}\right.$; see Sect. 3.1.2) is more than a factor of five higher than that expected to be lost by a lower mass star during the RSG phase $\left(<1 M_{\odot}\right.$ for a star with an initial mass of $16 M_{\odot}$; Beasor \& Davies 2018). As a consequence, there are a number of consistent, although not compelling, indications suggesting that the progenitor of SN 2015da was an LBV star.

\section{Summary and conclusions}

In this paper, we reported the main results of the photometric and spectroscopic follow-up of the Type IIn SN 2015da, exploded in the relatively nearby $(D \simeq 53.2 \mathrm{Mpc})$ host galaxy NGC 5337 .

SN 2015da is a long-lasting Type IIn SN discovered soon after explosion and with well-sampled photometric and spectroscopic follow-up from the optical to MIR wavelengths. This makes it one of the best followed SN IIn, with, to date, more than four years of collected data. Follow-up campaigns of this nearby optical transient are still ongoing both in the optical and NIR and MIR domains, and additional data could help us to better constrain the physical properties of the explosion, its dusty environment, and ultimately the nature of its progenitor star.

The transient exploded in a highly obscured environment, contributing a reddening of $E(B-V)=0.97 \mathrm{mag}\left(A_{V} \simeq 3 \mathrm{mag}\right.$ assuming a canonical extinction law with $R_{V}=3.1$ ) in the direction of SN 2015da. The IR excess observed from $+433 \mathrm{~d}$ suggests that the IR luminosity is most likely produced by radiatively heated dust. This conclusion is supported by the shape of the $\mathrm{H} \alpha$ line profiles, which show symmetric wings with respect to their centroids at all phases, while alternative explanations, like rapid formation of large dust grains at the interface between the forward and reverse shock, would result in strong asymmetries in the line shape. On the other hand, given the simplicity of the models adopted here and the assumptions made, we cannot definitely rule out any of the possible emission mechanisms.

The analysis of the pseudo-bolometric light curve of SN 2015da revealed an extended CSM with a total mass 5.5-10.3 $M_{\odot}$ (assuming an energy conversion efficiency $\epsilon=$ 0.25 ), while wind velocities suggest a very massive precursor, possibly an LBV, as a viable progenitor for SN 2015da. This conclusion is supported by the very high mass-loss rate inferred from the evolution of the pseudo-bolometric luminosity $(\dot{M} \simeq$ $\left.0.6-0.7 M_{\odot} \mathrm{yr}^{-1}\right)$, which indicates that multiple outbursts during a long-lasting eruptive phase, similar to those observed in other SN impostors such as SN 2000ch (Wagner et al. 2004; Pastorello et al. 2010) are responsible for the dense CSM surrounding the progenitor star at the time of its explosion. We note, however, that the upper CSM mass limit would require a progenitor that retained a massive envelope until the very last phases of its evolution. According to the Geneva stellar evolutionary 
models (Hirschi et al. 2004), this is problematic at solar metallicity. For example, a nonrotating $25 M_{\odot}$ star has an envelope mass of $\simeq 10 M_{\odot}$ at the point of explosion, while a more massive (or rotating) star would lose even more mass to then explode as a stripped-envelope SN. While this envelope mass is larger than the ejected CSM, it would require that the "entire" envelope was ejected just before explosion. If we assume that only some fraction of the envelope can be ejected immediately prior to $\mathrm{CC}$, we must turn to lower metallicity models, which, on the other hand, have commensurately smaller $\dot{M}$. On the other hand, even at SMC metallicity, it is hard to form a H-rich star that has an envelope mass exceeding $10 M_{\odot}$ at the time of CC. At even lower metallicity, for example, $Z=10^{-5}$, wind-driven mass loss is much smaller, and it is possible to form a progenitor with a ZAMS mass of $60 M_{\odot}$ that will explode as a Type II SN (Meynet \& Maeder 2002). However, while this was plausible for SN 2010jl that exploded in a faint irregular galaxy, NGC 5337 is a normal spiral, with a roughly solar metallicity (see Sect. 2). One possibility is that SN 2015da is hosted in a faint dwarf satellite of NGC 5337, which is by chance projected on the sky towards towards the main galaxy. Future spectroscopy at the site of SN 2015da after the SN has faded may allow us to measure the local metallicity, or, alternatively, to identify a kinematically distinct dwarf host. Without this information, the contradiction between the nearly solar metallicity host and the requirement for a low metallicity progenitor remains a puzzle.

Acknowledgements. We thank Marco Berton, Sina Chen, Fabio Briganti, Fabio Martinelli, Emmanuel Conseil, Stan Howerton, Masanori Mizutani and Kunihiro Shima for their help with the observations of SN 2015da. We are also grateful to our late friend Alex Dimai, whose observations have been used in this study. We gratefully acknowledge support from the Knut and Alice Wallenberg Foundation. The Oskar Klein Centre is funded by the Swedish Research Council. We acknowledge the support of the staff of the Xinglong $2.16 \mathrm{~m}$ telescope. This work was partially supported by the Open Project Program of the Key Laboratory of Optical Astronomy, National Astronomical Observatories, Chinese Academy of Sciences. M.F. is supported by a Royal Society - Science Foundation Ireland University Research Fellowship. J.H. acknowledges financial support from the Finnish Cultural Foundation and the Vilho, Yrjö and Kalle Väisälä Foundation of the Finnish Academy of Science and Letters. Research by D.J.S. is supported by NSF grants AST-1821987, AST-1821967, AST-1813708, AST-1813466 and AST-1908972. S.B., L.T.and M.T. are partially supported by the PRIN-INAF 2016 with the project "Towards the SKA and CTA era: discovery, localisation, and physics of transient sources" (PI: M. Giroletti). N E.-R. acknowledges support from the Spanish MICINN grant ESP2017-82674-R and FEDER funds D.A.H., C.M., and G.H. were supported by NSF AST-1313484 The work of X.W. is supported by the National Natural Science Foundation of China (NSFC grants 11325313, 11633002, and 11761141001), and the National Program on Key Research and Development Project (grant no. 2016YFA0400803). Research by S.V. is supported by NSF grant AST-1813176. J.Z. is supported by the National Natural Science Foundation of China (NSFC, grants 11773067, 11403096), the Youth Innovation Promotion Association of the CAS (grants 2018081), and the Western Light Youth Project. Based on observations collected at: ESO La Silla Observatory under program "Optical \& NIR monitoring of bright supernovae with REM" during AOT30. The Gemini Observatory, under program GN2016B-Q-57, which is operated by the Association of Universities for Research in Astronomy, Inc., under a cooperative agreement with the NSF on behalf of the Gemini partnership: the National Science Foundation (United States), the National Research Council (Canada), CONICYT (Chile), Ministerio de Ciencia, Tecnología e Innovación Productiva (Argentina), and Ministério da Ciência, Tecnologia e Inovação (Brazil). Tthe Nordic Optical Telescope, operated by the Nordic Optical Telescope Scientific Association and the Gran Telescopio Canarias (GTC), both installed at the Spanish Observatorio del Roque de los Muchachos of the Instituto de Astrofisica de Canarias, on the island of La Palma (Spain). The Copernico Telescope (Asiago, Italy) operated by INAF - Osservatorio Astronomico di Padova. The $3 \mathrm{~m}$ Shane Reflector, located at the Lick Observatory (7281 Mt Hamilton Rd, Mt Hamilton, CA 95140, USA.) owned and operated by the University of California. This work makes use of observations from the Las Cumbres Observatory network of telescopes. We acknowledge the support of the staff of the Li-Jiang $2.4 \mathrm{~m}$ telescope (LJT) Funding for the LJT has been provided by the Chinese Academy of Sciences (CAS) and the People's Government of Yunnan Province. The LJT is jointly operated and administrated by Yunnan Observatories and Center for Astronomical Mega-Science, CAS. This research has made use of the Keck Observatory Archive (KOA), which is operated by the W. M. Keck Observatory and the NASA Exoplanet Science Institute (NExScI), under contract with the National Aeronautics and Space Administration. Some of the data presented herein were obtained at the W. M. Keck Observatory, which is operated as a scientific partnership among the California Institute of Technology, the University of California and the National Aeronautics and Space Administration. The Observatory was made possible by the generous financial support of the W. M. Keck Foundation. The authors wish to recognize and acknowledge the very significant cultural role and reverence that the summit of Mauna Kea has always had within the indigenous Hawaiian community. We are most fortunate to have the opportunity to conduct observations from this mountain. This publication makes use of data products from NEOWISE, which is a project of the Jet Propulsion Laboratory/California Institute of Technology, founded by the Planetary Science Division of the National Aeronautics and Space Administration. This research has made use of the Keck Observatory Archive (KOA), which is operated by the W. M. Keck Observatory and the NASA Exoplanet Science Institute (NExScI), under contract with the National Aeronautics and Space Administration. This research has made use of the NASA/IPAC Extragalactic Database (NED) which is operated by the Jet Propulsion Laboratory, California Institute of Technology, under contract with the National Aeronautics and Space Administration. This research has made use of the NASA/IPAC Infrared Science Archive, which is operated by the Jet Propulsion Laboratory, California Institute of Technology, under contract with the National Aeronautics and Space Administration. This publication makes use of data products from the Two Micron All Sky Survey, which is a joint project of the University of Massachusetts and the Infrared Processing and Analysis Center/California Institute of Technology, funded by the National Aeronautics and Space Administration and the National Science Foundation. Funding for the Sloan Digital Sky Survey (SDSS) has been provided by the Alfred P. Sloan Foundation, the Participating Institutions, the National Aeronautics and Space Administration, the National Science Foundation, the U.S. Department of Energy, the Japanese Monbukagakusho, and the Max Planck Society. The SDSS Web site is http://www.sdss.org/. This publication makes use of data products from the Wide-field Infrared Survey Explorer, which is a joint project of the University of California, Los Angeles, and the Jet Propulsion Laboratory/California Institute of Technology, funded by the National Aeronautics and Space Administration. The SDSS is managed by the Astrophysical Research Consortium (ARC) for the Participating Institutions. The Participating Institutions are The University of Chicago, Fermilab, the Institute for Advanced Study, the Japan Participation Group, The Johns Hopkins University, Los Alamos National Laboratory, the Max-Planck-Institute for Astronomy (MPIA), the Max-Planck-Institute for Astrophysics (MPA), New Mexico State University, University of Pittsburgh, Princeton University, the United States Naval Observatory, and the University of Washington. The intermediate Palomar Transient Factory project is a scientific collaboration among the California Institute of Technology, Los Alamos National Laboratory, the University of Wisconsin, Milwaukee, the Oskar Klein Center, the Weizmann Institute of Science, the TANGO Program of the University System of Taiwan, and the Kavli Institute for the Physics and Mathematics of the Universe. IRAF is distributed by the National Optical Astronomy Observatory, which is operated by the Association of Universities for Research in Astronomy (AURA) under a cooperative agreement with the National Science Foundation. SNOOPY is a package for SN photometry using PSF fitting and/or template subtraction developed by E. Cappellaro. A package description can be found at http:// sngroup.oapd.inaf.it/snoopy.html. FOSCGUI is a graphic user interface aimed at extracting SN spectroscopy and photometry obtained with FOSC-like instruments. It was developed by E. Cappellaro. A package description can be found at http://sngroup.oapd.inaf.it/foscgui.html.

\section{References}

Adelman-McCarthy, J. K., Agüeros, M. A., Allam, S. S., et al. 2006, ApJS, 162, 38

Agnoletto, I., Benetti, S., Cappellaro, E., et al. 2009, ApJ, 691, 1348

Aldering, G., Antilogus, P., Bailey, S., et al. 2006, ApJ, 650, 510

Alloin, D., Collin-Souffrin, S., Joly, M., \& Vigroux, L. 1979, A\&A, 78, 200

Andrews, J. E., Clayton, G. C., Wesson, R., et al. 2011, AJ, 142, 45

Asplund, M., Grevesse, N., Sauval, A. J., \& Scott, P. 2009, ARA\&A, 47, 481

Balberg, S., \& Loeb, A. 2011, MNRAS, 414, 1715

Beasor, E. R., \& Davies, B. 2018, MNRAS, 475, 55

Benetti, S., Cappellaro, E., Turatto, M., et al. 2006, ApJ, 653, L129

Bohlin, R. C., Savage, B. D., \& Drake, J. F. 1978, ApJ, 224, 132

Borish, H. J., Huang, C., Chevalier, R. A., et al. 2015, ApJ, 801, 7

Botticella, M. T., Smartt, S. J., Kennicutt, R. C., et al. 2012, A\&A, 537, A132

Bottinelli, L., Gouguenheim, L., Paturel, G., \& Teerikorpi, P. 1995, A\&A, 296, 64 
Brown, T. M., Baliber, N., Bianco, F. B., et al. 2013, PASP, 125, 1031 Cardelli, J. A., Clayton, G. C., \& Mathis, J. S. 1989, ApJ, 345, 245

Chevalier, R. A. 1982a, ApJ, 258, 790

Chevalier, R. A. 1982b, ApJ, 259, 302

Chevalier, R. A., \& Fransson, C. 1994, ApJ, 420, 268

Chevalier, R. A., \& Fransson, C. 2017, Thermal and Non-thermal Emission from Circumstellar Interaction (Berlin: Springer), 875

Chevalier, R. A., \& Irwin, C. M. 2011, ApJ, 729, L6

Chonis, T. S., \& Gaskell, C. M. 2008, AJ, 135, 264

Chugai, N. N. 2001, MNRAS, 326, 1448

Deng, J., Kawabata, K. S., Ohyama, Y., et al. 2004, ApJ, 605, L37

Denicoló, G., Terlevich, R., \& Terlevich, E. 2002, MNRAS, 330, 69

Dessart, L., Audit, E., \& Hillier, D. J. 2015, MNRAS, 449, 4304

Di Carlo, E., Massi, F., Valentini, G., et al. 2002, ApJ, 573, 144

Dilday, B., Howell, D. A., Cenko, S. B., et al. 2012, Science, 337, 942

Draine, B. T., \& Lee, H. M. 1984, ApJ, 285, 89

Dwek, E. 1983, ApJ, 274, 175

Dwek, E. 1985, ApJ, 297, 719

Elias-Rosa, N., Benetti, S., Cappellaro, E., et al. 2006, MNRAS, 369, 1880

Faber, S. M., Phillips, A. C., Kibrick, R. I., et al. 2003, Proc. SPIE, 4841, 1657

Ferlet, R., Vidal-Madjar, A., \& Gry, C. 1985, ApJ, 298, 838

Foley, R. J., Smith, N., Ganeshalingam, M., et al. 2007, ApJ, 657, L105

Fox, O., Skrutskie, M. F., Chevalier, R. A., et al. 2009, ApJ, 691, 650

Fox, O. D., Chevalier, R. A., Dwek, E., et al. 2010, ApJ, 725, 1768

Fox, O. D., Chevalier, R. A., Skrutskie, M. F., et al. 2011, ApJ, 741, 7

Fox, O. D., Filippenko, A. V., Skrutskie, M. F., et al. 2013, AJ, 146, 2

Fox, O. D., Silverman, J. M., Filippenko, A. V., et al. 2015a, MNRAS, 447, 772

Fox, O. D., Smith, N., Ammons, S. M., et al. 2015b, MNRAS, 454, 4366

Fransson, C. 1984, A\&A, 133, 264

Fransson, C., Chevalier, R. A., Filippenko, A. V., et al. 2002, ApJ, 572, 350

Fransson, C., Challis, P. M., Chevalier, R. A., et al. 2005, ApJ, 622, 991

Fransson, C., Ergon, M., Challis, P. J., et al. 2014, ApJ, 797, 118

Fremling, C., Sollerman, J., Taddia, F., et al. 2016, A\&A, 593, A68

Gal-Yam, A. 2019, ARA\&A, 57, 305

Gal-Yam, A., \& Leonard, D. C. 2009, Nature, 458, 865

Gall, C., Hjorth, J., Watson, D., et al. 2014, Nature, 511, 326

Garavini, G., Folatelli, G., Goobar, A., et al. 2004, AJ, 128, 387

Gerardy, C. L., Fesen, R. A., Nomoto, K., et al. 2002, ApJ, 575, 1007

González, M., Audit, E., \& Huynh, P. 2007, A\&A, 464, 429

Graham, J. R., \& Meikle, W. P. S. 1986, MNRAS, 221, 789

Hakobyan, A. A., Mamon, G. A., Petrosian, A. R., Kunth, D., \& Turatto, M. 2009, A\&A, 508, 1259

Hamuy, M., Phillips, M. M., Suntzeff, N. B., et al. 2003, Nature, 424, 651

Herbig, G. H. 1995, ARA\&A, 33, 19

Hirschi, R., Meynet, G., \& Maeder, A. 2004, A\&A, 425, 649

Hsiao, E. Y., Phillips, M. M., Marion, G. H., et al. 2019, PASP, 131, 014002

Huang, C., \& Chevalier, R. A. 2018, MNRAS, 475, 1261

Inserra, C., Fraser, M., Smartt, S. J., et al. 2016, MNRAS, 459, 2721

Jura, M., \& Kleinmann, S. G. 1990, ApJS, 73, 769

Kankare, E., Ergon, M., Bufano, F., et al. 2012, MNRAS, 424, 855

Katz, B., Sapir, N., \& Waxman, E. 2012, ApJ, 747, 147

Kiewe, M., Gal-Yam, A., Arcavi, I., et al. 2012, ApJ, 744, 10

Kotak, R., Meikle, W. P. S., Adamson, A., et al. 2004, MNRAS, 354, L13

Laor, A., \& Draine, B. T. 1993, ApJ, 402, 441

Li, W., Leaman, J., Chornock, R., et al. 2011, VizieR Online Data Catalog: VII/41

López-Sánchez, Á. R., Dopita, M. A., Kewley, L. J., et al. 2012, MNRAS, 426, 2630

Mattila, S., Meikle, W. P. S., Lundqvist, P., et al. 2008, MNRAS, 389, 141

Mauerhan, J. C., Smith, N., Silverman, J. M., et al. 2013, MNRAS, 431, 2599

Maund, J. R., Smartt, S. J., Kudritzki, R. P., et al. 2006, MNRAS, 369, 390

Mauron, N., \& Josselin, E. 2011, A\&A, 526, A156

Meynet, G., \& Maeder, A. 2002, A\&A, 390, 561

Moriya, T. J. 2014, A\&A, 564, A83

Morris, P. W., Waters, L. B. F. M., Barlow, M. J., et al. 1999, Nature, 402, 502

Nyholm, A., Sollerman, J., Tartaglia, L., et al. 2020, A\&A, accepted https://doi .org/10.1051/0004-6361/201836097

Ofek, E. O., Cameron, P. B., Kasliwal, M. M., et al. 2007, ApJ, 659, L13

Ofek, E. O., Rabinak, I., Neill, J. D., et al. 2010, ApJ, 724, 1396

Ofek, E. O., Arcavi, I., Tal, D., et al. 2014a, ApJ, 788, 154

Ofek, E. O., Zoglauer, A., Boggs, S. E., et al. 2014b, ApJ, 781, 42

Osterbrock, D. E., \& Ferland, G. J. 2006, Astrophysics of Gaseous Nebulae and Active Galactic Nuclei (USA: University Science Books)

Pastorello, A., Aretxaga, I., Zampieri, L., Mucciarelli, P., \& Benetti, S. 2005, ASP Conf. Ser., 342, 285

Pastorello, A., Smartt, S. J., Mattila, S., et al. 2007, Nature, 447, 829
Pastorello, A., Mattila, S., Zampieri, L., et al. 2008, MNRAS, 389, 113 Pastorello, A., Botticella, M. T., Trundle, C., et al. 2010, MNRAS, 408, 181 Petropoulou, M., Coenders, S., Vasilopoulos, G., Kamble, A., \& Sironi, L. 2017, MNRAS, 470, 1881

Pettini, M., \& Pagel, B. E. J. 2004, MNRAS, 348, L59

Pilyugin, L. S., Vílchez, J. M., \& Contini, T. 2004, A\&A, 425, 849 Poznanski, D., Prochaska, J. X., \& Bloom, J. S. 2012, MNRAS, 426, 1465

Pozzo, M., Meikle, W. P. S., Fassia, A., et al. 2004, MNRAS, 352, 457

Schlafly, E. F., \& Finkbeiner, D. P. 2011, ApJ, 737, 103

Schlegel, E. M. 1990, MNRAS, 244, 269

Silverman, J. M., Nugent, P. E., Gal-Yam, A., et al. 2013, ApJS, 207, 3

Skrutskie, M. F., Cutri, R. M., Stiening, R., et al. 2006, AJ, 131, 1163

Smith, N., \& Frew, D. J. 2011, MNRAS, 415, 2009

Smith, N., Gehrz, R. D., Hinz, P. M., et al. 2003, AJ, 125, 1458

Smith, N., Li, W., Foley, R. J., et al. 2007, ApJ, 666, 1116

Smith, N., Hinkle, K. H., \& Ryde, N. 2009a, AJ, 137, 3558

Smith, N., Silverman, J. M., Chornock, R., et al. 2009b, ApJ, 695, 1334

Smith, N., Miller, A., Li, W., et al. 2010, AJ, 139, 1451

Smith, N., Li, W., Silverman, J. M., Ganeshalingam, M., \& Filippenko, A. V. 2011, MNRAS, 415, 773

Smith, N., Silverman, J. M., Filippenko, A. V., et al. 2012, AJ, 143, 17

Sollerman, J., Cox, N., Mattila, S., et al. 2005, A\&A, 429, 559

Stathakis, R. A., \& Sadler, E. M. 1991, MNRAS, 250, 786

Stoll, R., Prieto, J. L., Stanek, K. Z., et al. 2011, ApJ, 730, 34

Stritzinger, M., Taddia, F., Fransson, C., et al. 2012, ApJ, 756, 173

Sutherland, R. S., \& Dopita, M. A. 2017, ApJS, 229, 34

Suzuki, N., \& Fukugita, M. 2018, AJ, 156, 219

Svirski, G., Nakar, E., \& Sari, R. 2012, ApJ, 759, 108

Taddia, F., Sollerman, J., Razza, A., et al. 2013a, A\&A, 558, A143

Taddia, F., Stritzinger, M. D., Sollerman, J., et al. 2013b, A\&A, 555, A10

Taddia, F., Sollerman, J., Fremling, C., et al. 2015, A\&A, 580, A131

Tartaglia, L., Pastorello, A., Taubenberger, S., et al. 2015, MNRAS, 447, 117

Tartaglia, L., Elias-Rosa, N., Pastorello, A., et al. 2016, ApJ, 823, L23

Tartaglia, L., Sand, D. J., Valenti, S., et al. 2018, ApJ, 853, 62

Tomasella, L., Cappellaro, E., Pumo, M. L., et al. 2018, MNRAS, 475, 1937

Trundle, C., Kotak, R., Vink, J. S., et al. 2008, A\&A, 483, L47

Tsuna, D., Kashiyama, K., \& Shigeyama, T. 2019, ApJ, 884, 87

Tully, R. B., Courtois, H. M., \& Sorce, J. G. 2016, AJ, 152, 50

Turatto, M., Cappellaro, E., Danziger, I. J., et al. 1993, MNRAS, 262, 128

van Driel, W., Marcum, P., Gallagher, III, J. S., et al. 2001, A\&A, 378, 370

Van Dyk, S. D., Peng, C. Y., King, J. Y., et al. 2000, PASP, 112, 1532

Vink, J. S. 2012, Astrophys. Space Sci. Lib., 384, 221

Vlasis, A., Dessart, L., \& Audit, E. 2016, MNRAS, 458, 1253

Wagner, R. M., Vrba, F. J., Henden, A. A., et al. 2004, PASP, 116, 326

Wang, L., Baade, D., Höflich, P., et al. 2004, ApJ, 604, L53

Wegner, G., \& Swanson, S. R. 1996, MNRAS, 278, 22

Wood-Vasey, W. M., Wang, L., \& Aldering, G. 2004, ApJ, 616, 339

Wright, E. L. 1980, ApJ, 242, L23

Yaron, O., \& Gal-Yam, A. 2012, PASP, 124, 668

Zhang, J. \& Wang, X. 2015, ATel, 6939

Zhang, T., Wang, X., Wu, C., et al. 2012, AJ, 144, 131

${ }^{1}$ Department of Astronomy and the Oskar Klein Centre, Stockholm University, AlbaNova, Roslagstullsbacken 21, 11421 Stockholm, Sweden

e-mail: leonardo.tartaglia@astro.su.se

2 INAF - Osservatorio Astronomico di Padova, Vicolo dell'Osservatorio 5, 35122 Padova, Italy

3 Tuorla Observatory, Department of Physics and Astronomy, 20014, University of Turku, Turku, Finland

4 School of Physics, O'Brien Centre for Science North, University College Dublin, Belfield Dublin 4, Ireland

5 Department of Applied Physics, Universidad de Cádiz, campus of Puerto Real, 11510 Cádiz, Spain

${ }^{6}$ Institute of Space Sciences (ICE, CSIC), Campus UAB, Camí de Can Magrans s/n, 08193, Cerdanyola del Vallès, Barcelona, Spain

7 Institut d'Estudis Espacials de Catalunya (IEEC), c/Gran Capità 24, Edif. Nexus 201, 08034 Barcelona, Spain

8 Benoziyo Center for Astrophysics and the Helen Kimmel center for planetary science, Weizmann Institute of Science, 76100 Rehovot, Israel

9 Xinjiang Astronomical Observatory, 150 Science 1-Street, Urumqi, Xinjiang 830011, PR China 
${ }^{10}$ Xingming Observatory, Mountain Nanshan, Urumqi, Xinjiang 830011, PR China

11 Graduate Institute of Astronomy, National Central University, 300 Zhongda Rd., Zhongli District, Taoyuan City 32001, Taiwan, PR China

12 Department of Astronomy/Steward Observatory, 933 North Cherry Avenue, Rm. N204, Tucson, AZ 85721-0065, USA

13 Osservatorio Astronomico di Monte Agliale, Via Cune Motrone, 55023 Borgo a Mozzano, Lucca, Italy

14 Physics Department and Tsinghua Center for Astrophysics, Tsinghua University, Beijing 100084, PR China

15 Yunnan Observatories, Chinese Academy of Sciences, Kunming 650216, PR China

${ }^{16}$ Key Laboratory for the Structure and Evolution of Celestial Objects, Chinese Academy of Sciences, Kunming 650216, PR China

17 Department of Astronomy, School of Physics and Astronomy, Shanghai Jiaotong Univeristy, Shanghai 200240, PR China

18 Key Laboratory of Optical Astronomy, National Astronomical Observatories, Chinese Academy of Sciences, 10101 Beijing, PR China
${ }^{19}$ School of Astronomy and Space Science, University of Chinese Academy of Sciences, 101408 Beijing, PR China

${ }^{20}$ Center for Astrophysics I Harvard \& Smithsonian, 60 Garden Street, Cambridge, MA 02138-1516, USA

${ }^{21}$ Las Cumbres Observatory, 6740 Cortona Drive, Suite 102, Goleta, CA 93117-5575, USA

22 Department of Physics, University of California, Santa Barbara, CA 93106-9530, USA

23 Department of Physics, University of California, Davis, CA 95616, USA

24 The Oskar Klein Centre, Physics Department, Stockholm University, AlbaNova, Roslagstullsbacken 21, 21 Stockholm, Sweden

25 Division of Physics, Mathematics and Astronomy, California Institute of Technology, Pasadena, CA 91125, USA

${ }^{26}$ Space Telescope Science Institute, 3700 San Martin Drive, Baltimore, MD 21218, USA

27 European Southern Observatory Karl - Schwarzschild - Str 285748 , Garching bei München, Germany

${ }^{28}$ Center for Interdisciplinary Exploration and Research in Astrophysics (CIERA) and Department of Physics and Astronomy, Northwestern University, Evanston, IL 60208, USA 


\section{Appendix A: Observations and data reduction}

The follow-up campaign of SN 2015da spanned a period of more than $1500 \mathrm{~d}$ after the SN explosion and involved a number of collaborations and facilities. The names of the telescopes and instruments used are reported in Tables A.1-A.4 (available at the CDS), and A.6.

\section{A.1. Photometric data}

Optical photometric data were mainly obtained using the telescopes of the Las Cumbres Observatory ${ }^{18}$ network (Brown et al. 2013) within the Supernova Key Project, while most of the NIR data and additional optical photometry were provided by the NUTS collaboration ${ }^{19}$, using the $2.56 \mathrm{~m}$ Nordic Optical Telescope (NOT, at the Observatorio del Roque de los Muchacos, La Palma, Spain) with NOTCam and ALFOSC. Optical data were also collected using the $1.82 \mathrm{~m}$ Copernico Telescope (at the INAF Osservatorio Astronomico di Asiago, Italy) with AFOSC and iPTF, the automated Mount Palomar 60 inch (P60) and the 48 inch Samuel Oschin (P48) telescopes. The iPTF survey did not monitor this part of the sky at all in 2015, so the transient was only detected by iPTF in March 2016, which is the reason why it was internally dubbed iPTF16tu. iPTF then followed the object for a full year until the end of the survey. Early data covering the rise to maximum light and a few additional epochs were provided by amateurs and calibrated to the $R$ band. These data helped constrain the explosion epoch and the rise of SN 2015da (see Sect. 3.1).

NIR photometry was almost entirely provided by NUTS using the NOT with NOTCam, while early data around maximum were provided by the ESO $0.6 \mathrm{~m}$ Rapid Eye Mount telescope in La Silla (Chile). One additional NIR epoch was obtained using the Near Infrared Camera Spectrometer $\left(\mathrm{NICS}^{20}\right)$ mounted at the $3.58 \mathrm{~m}$ Telescopio Nazionale Galileo (TNG) located at the Observatorio del Roque de los Muchachos in La Palma (Spain).

Optical and NIR pre-reduction steps were performed using standard IRAF tasks. NOTCam frames pre-reduced using an adapted version of the external IRAF package NOTCAM ${ }^{21}$, using bad pixel masking, differential flat-fielding method, sky subtraction, distortion correction, and stacking of dithered images. Final magnitudes were mostly obtained using the SuperNOva PhotometrY (SNOOPY ${ }^{22}$ ) pipeline and calibrated on a local sequence of stars obtained through the Sloan Digital Sky Survey $\left(\operatorname{SDSS}^{23}\right.$ ) (for the optical frames) and the Two Micron All Sky Survey (2MASS ${ }^{24}$ ) catalogs. UBVRI magnitudes of the reference stars were obtained transforming the SDSS magnitudes following Chonis \& Gaskell (2008). Magnitudes of the local standard stars are provided in Table A.5. P60 data were reduced using the dedicated pipeline described in Fremling et al. (2016).
MIR magnitudes were obtained as detailed below. For every pass, after a quick check to verify that the $\mathrm{SN}$ did not show rapid variations, all high-quality images (obtained typically within 3-5 d) available in the NEOWISE 2019 Data Release have been co-added ${ }^{25}$ and retrieved. The $\mathrm{SN}$ exploded in a region in which the galaxy background was still significant, while the spatial resolution was relatively poor ( $\geq 6$ arcsec). We therefore decided to apply the galaxy background subtraction method. To this aim, we use the averages of all images acquired during the year 2014, meaning before explosion, as background in both bands. After accurate re-entering, the images were ready to be subtracted, satisfactorily removing the galaxy, and leaving the $\mathrm{SN}$ alone. On the subtraction image we performed aperture photometry with aperture of eight and nine pixels. We then applied the aperture correction determined by applying the same aperture on a number of nearby, isolated reference stars of the same magnitude of the SN, present in the AllWISE Source Catalog. Following these prescriptions, the $W_{1}$ and $W_{2}$ magnitudes of SN 2015da reported in Table A.4 are on the same scale as the AllWISE catalog.

\section{A.2. Spectroscopic data}

The spectroscopic follow-up campaign was carried out mostly using the NOT with ALFOSC and the $1.82 \mathrm{~m}$ Copernico Telescope with AFOSC, and the data were reduced using the dedicated pipeline FOSCGUI ${ }^{26}$. Three additional spectra were obtained using the $2 \mathrm{~m}$ Faulkes Telescope North (FTN) telescope with FLOYDS of the Las Cumbres Observatory network. Spectra at different phases were provided by the TNG with DOLORes, the $10.4 \mathrm{~m}$ Gran Telescopio Canarias (GTC, located at the Observatorio del Roque de los Muchachos in La Palma) with OSIRIS, the $3 \mathrm{~m}$ Donald Shane Telescope (at the Lick Observatory in San Jose, California, USA) with KAST and the 200 inch Hale telescope with DBSP at the Mount Palomar Observatory (San Diego, California, USA). All these spectra were reduced using standard IRAF tasks. The classification spectrum was obtained using the Lijiang $2.4 \mathrm{~m}$ telescope (LJT) at the Lijiang Observatory of Yunnan Observatories with YFOSC, which also provided three early-phase spectra $(+11,+19$ and $+26 \mathrm{~d}$ after explosion). Two additional early-phase spectra $(+10$ and $+36 \mathrm{~d}$ ) were obtained using the ZTA $2.6 \mathrm{~m}$ telescope located at the Byurakan Astrophysical Observatory (BAO, Armenia) with XLT. All these spectra were reduced using standard IRAF tasks. NIR spectra were provided by the TNG using NICS and reduced using IRAF tasks, and the $8.1 \mathrm{~m}$ Gemini North telescope (located at the Mauna Kea Observatory, Hawaii, USA) using the Gemini Near-InfraRed Spectrograph (GNIRS), reduced as in Hsiao et al. (2019). A complete set of optical spectra is shown in Fig. A.1.

\footnotetext{
${ }^{18}$ https://lco.global/

19 http://csp2.lco.cl/not/

${ }^{20}$ http://www.tng.iac.es/instruments/nics/

${ }^{21}$ v.2.5; http://www.not.iac.es/instruments/notcam/guide/ observe.html\#reductions

22 http://sngroup.oapd.inaf.it/snoopy.html

23 http://www.sdss.org/

24 https://www.ipac. caltech.edu/2mass/
}

${ }^{25}$ With the WISE/NEOWISE Co-adder https://irsa.ipac. caltech.edu/applications/ICORE/.

${ }^{26}$ http://sngroup. oapd.inaf.it/foscgui .html 


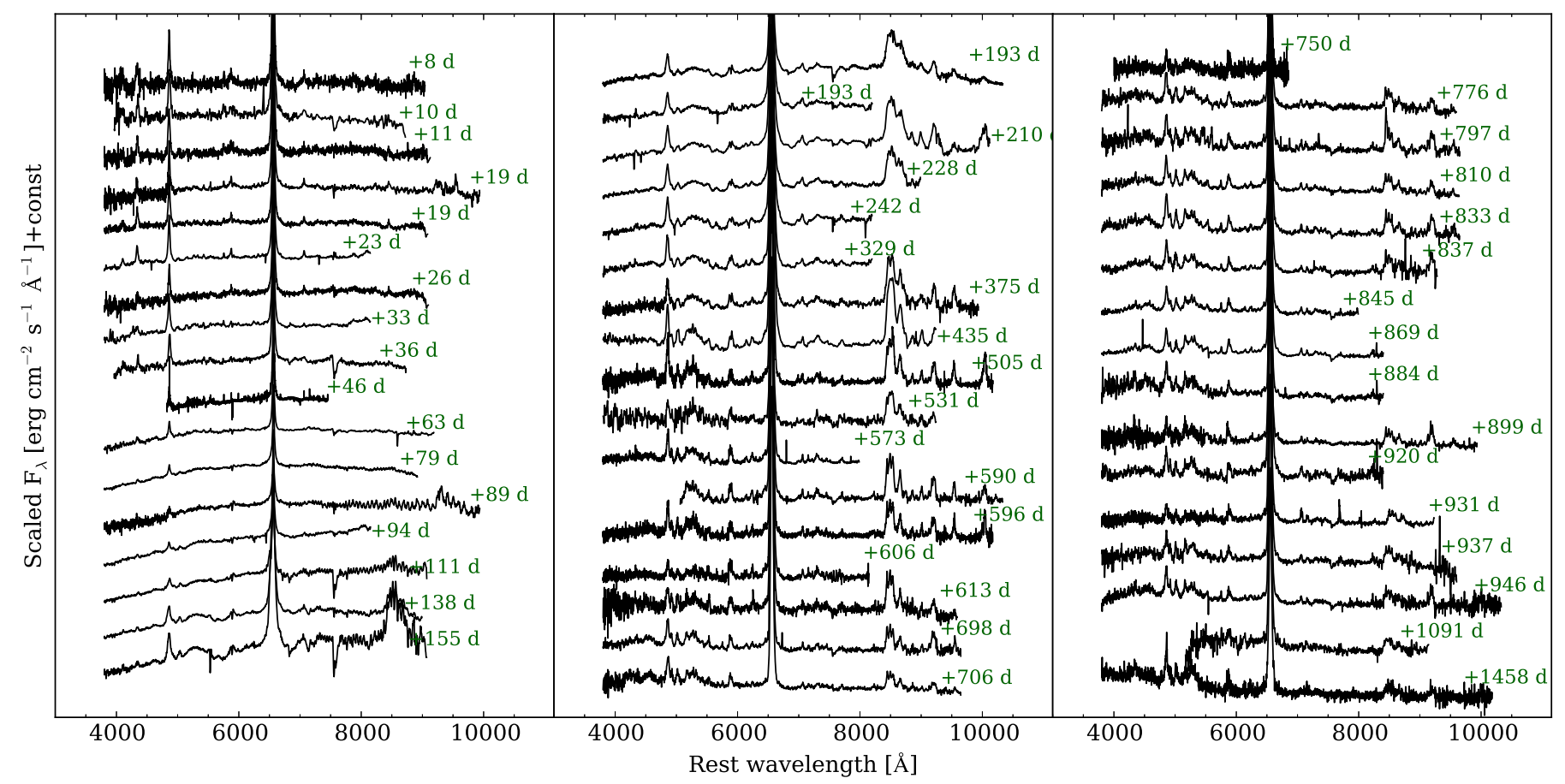

Fig. A.1. Complete set of optical spectroscopic observations of SN 2015da. Spectra were not corrected for extinction to facilitate the comparison at wavelengths bluer than $\simeq 5000 \AA$. Phases refer to the estimated epoch of the explosion.

Table A.5. Local sequence of stars used for the optical and NIR photometric calibration.

\begin{tabular}{|c|c|c|c|c|c|c|c|c|c|c|c|c|c|c|}
\hline $\begin{array}{l}\text { RA [J2000] } \\
\text { (hh:mm:ss) }\end{array}$ & $\begin{array}{l}\text { Dec [J2000] } \\
\text { (dd:mm:ss) }\end{array}$ & $\begin{array}{l}u \text { (err) } \\
\text { (mag) }\end{array}$ & $\begin{array}{l}g(\mathrm{err}) \\
(\mathrm{mag})\end{array}$ & $\begin{array}{l}r \text { (err) } \\
\text { (mag) }\end{array}$ & $\begin{array}{l}i \text { (err) } \\
\text { (mag) }\end{array}$ & $\begin{array}{l}z \text { (err) } \\
\text { (mag) }\end{array}$ & $\begin{array}{l}U \text { (err) } \\
\text { (mag) }\end{array}$ & $\begin{array}{l}B \text { (err) } \\
\text { (mag) }\end{array}$ & $\begin{array}{l}V \text { (err) } \\
\text { (mag) }\end{array}$ & $\begin{array}{l}R \text { (err) } \\
\text { (mag) }\end{array}$ & $\begin{array}{l}I \text { (err) } \\
\text { (mag) }\end{array}$ & $\begin{array}{l}J \text { (err) } \\
\text { (mag) }\end{array}$ & $\begin{array}{c}H \text { (err) } \\
\text { (mag) }\end{array}$ & $\begin{array}{l}K \text { (err) } \\
(\mathrm{mag})\end{array}$ \\
\hline :52:08.58 & $+39: 43: 33.78$ & - & - & - & - & - & - & - & - & - & - & $16.03(0.07)$ & $15.17(0.09)$ & $14.91(0.10)$ \\
\hline 13:52:09.85 & $+39: 38: 56.11$ & - & - & - & - & - & - & - & - & - & - & $15.44(0.05)$ & $14.91(0.06)$ & $14.75(0.09)$ \\
\hline $13: 52: 12.32$ & $+39: 42: 37.14$ & $21.15(0.07)$ & $18.67(0.02)$ & $17.51(0.02)$ & $17.02(0.02)$ & $16.71(0.02)$ & $20.30(0.07)$ & $19.26(0.07)$ & $17.98(0.04)$ & $17.22(0.05)$ & $16.49(0.11)$ & $15.44(0.04)$ & $14.82(0.06)$ & $14.66(0.09)$ \\
\hline $13: 52: 15.65$ & $+39: 40: 02.69$ & $17.39(0.02)$ & $16.47(0.02)$ & $16.26(0.01)$ & $16.17(0.02)$ & $16.17(0.02)$ & $16.53(0.02)$ & $16.76(0.04)$ & $16.34(0.03)$ & $16.08(0.03)$ & $15.77(0.05)$ & $15.40(0.04)$ & $15.19(0.08)$ & $14.88(0.10)$ \\
\hline $13: 52: 17.43$ & $+39: 40: 56.13$ & $16.68(0.02)$ & $14.68(0.02)$ & $13.80(0.01)$ & $13.411(0.001)$ & $13.22(0.02)$ & $15.83(0.02)$ & $15.18(0.05)$ & $14.15(0.03)$ & $13.54(0.04)$ & $12.91(0.09)$ & $12.15(0.02)$ & $11.55(0.02)$ & $11.46(0.02)$ \\
\hline $13: 52: 20.88$ & $+39: 44: 19.68$ & - & - & - & - & - & - & - & - & - & - & $12.78(0.02)$ & $12.24(0.03)$ & $12.13(0.03)$ \\
\hline $13: 52: 26.97$ & $+39: 39: 55.39$ & $21.02(0.08)$ & $19.19(0.02)$ & $18.45(0.02)$ & $18.14(0.02)$ & $18.01(0.02)$ & $20.17(0.08)$ & $19.65(0.05)$ & $18.75(0.03)$ & $18.21(0.04)$ & $17.67(0.07)$ & - & - & - \\
\hline $13: 52: 28.08$ & $+39: 39: 14.57$ & $21.21(0.09)$ & $18.85(0.01)$ & $17.61(0.02)$ & $17.10(0.02)$ & $16.81(0.02)$ & $20.35(0.09)$ & $19.47(0.07)$ & 18.11(0.04) & $17.31(0.05)$ & $16.55(0.11)$ & $15.69(0.05)$ & $15.03(0.05)$ & $14.86(0.09)$ \\
\hline $13: 52: 29.22$ & $+39: 41: 02.24$ & $19.99(0.04)$ & $17.66(0.01)$ & $16.60(0.02)$ & $16.19(0.02)$ & $15.94(0.02)$ & $19.13(0.04)$ & $18.22(0.06)$ & $17.03(0.03)$ & $16.33(0.05)$ & $15.68(0.09)$ & $14.83(0.03)$ & $14.19(0.04)$ & $14.12(0.05)$ \\
\hline $13: 52: 29.52$ & $+39: 41: 24.69$ & $17.86(0.02)$ & $16.35(0.02)$ & $15.83(0.02)$ & $15.62(0.02)$ & $15.58(0.02)$ & $17.01(0.02)$ & $16.74(0.04)$ & $16.03(0.03)$ & $15.61(0.03)$ & $15.18(0.06)$ & $14.74(0.03)$ & $14.44(0.04)$ & $14.30(0.06)$ \\
\hline $13: 52: 29.64$ & $+39: 39: 56.96$ & $15.28(0.02)$ & $13.97(0.01)$ & $14.286(0.001)$ & $13.49(0.02)$ & $13.49(0.01)$ & $14.42(0.02)$ & $14.08(0.03)$ & $14.14(0.02)$ & $13.91(0.08)$ & $12.86(0.16)$ & $12.70(0.02)$ & $12.40(0.02)$ & $12.37(0.02)$ \\
\hline $13: 52: 32.03$ & $+39: 39: 17.95$ & - & - & - & - & - & - & - & - & - & - & $15.76(0.06)$ & $15.04(0.07)$ & $14.81(0.09)$ \\
\hline $13: 52: 35.03$ & $+39: 41: 22.91$ & $21.52(0.08)$ & $19.59(0.02)$ & $18.48(0.02)$ & $18.13(0.02)$ & $17.66(0.02)$ & $20.67(0.08)$ & $20.17(0.06)$ & 18.93(0.04) & $18.23(0.04)$ & $17.64(0.08)$ & $15.72(0.08)$ & $14.96(0.08)$ & $14.38(0.08)$ \\
\hline $13: 52: 39.33$ & $+39: 43: 43.11$ & $18.14(0.02)$ & $16.86(0.02)$ & $16.41(0.02)$ & $16.26(0.02)$ & $16.23(0.02)$ & $17.29(0.02)$ & $17.23(0.04)$ & $16.59(0.03)$ & $16.21(0.03)$ & $15.84(0.06)$ & - & - & - \\
\hline
\end{tabular}

Notes. $U B V R I, J H K$ and $W_{1} W_{2}$ magnitudes are calibrated in the Vega photometric system, ugriz in the AB photometric system using values reported in the SDSS database. 
Table A.6. Log of the optical spectroscopic observations of SN 2015da.

\begin{tabular}{|c|c|c|c|c|c|c|c|}
\hline Date & JD & $\begin{array}{l}\text { Phase } \\
\text { (d) }\end{array}$ & Instrumental setup & Grism/grating & $\begin{array}{c}\text { Spectral range } \\
(\AA)\end{array}$ & $\begin{array}{c}\text { Resolution } \\
(\AA)\end{array}$ & $\begin{array}{c}\text { Exposure time } \\
\text { (s) }\end{array}$ \\
\hline 20150116 & 2457039.37 & +8 & GMG24+YFOSC/YSU & gr3 & $3470-9110$ & 15.95 & 1560 \\
\hline 20150118 & 2457041.37 & +10 & BAO $2.16 \mathrm{~m}+\mathrm{XLT}$ & 14.00 & $3970-8720$ & 15.00 & 3600 \\
\hline 20150119 & 2457042.38 & +11 & GMG24+YFOSC/YSU & gr3 & 3470-9130 & 20.86 & 1800 \\
\hline 20150127 & 2457049.99 & +19 & FTN+FLOYDS & $2351 / \mathrm{mm}$ & 3180-9940 & 14.08 & 3600 \\
\hline 20150127 & 2457050.37 & +19 & GMG24+YFOSC/YSU & gr3 & $3470-9080$ & 15.97 & 2100 \\
\hline 20150131 & 2457053.67 & +23 & Ekar $1.82 \mathrm{~m}+\mathrm{AFOSC}$ & gr4 & $3330-8150$ & 15.20 & 1200 \\
\hline 20150203 & 2457056.79 & +26 & TNG+NICS & IJ & $9000-14000$ & $26.86^{(\star \star)}$ & $4 \times 150$ \\
\hline 20150203 & 2457057.34 & +26 & GMG24+YFOSC/YSU & gr3 & $3480-9100$ & 15.20 & 1800 \\
\hline 20150210 & 2457063.55 & +33 & Ekar182+AFOSC & gr4 & $3330-8150$ & 14.83 & 1800 \\
\hline 20150213 & 2457067.35 & +36 & BAO $2.16 \mathrm{~m}+\mathrm{XLT}$ & 14.00 & $3960-8730$ & 15.00 & 2700 \\
\hline 20150223 & 2457077.16 & +46 & Keck+DEIMOS & 1200G/6170 & $4830-7460$ & 1.56 & $300+300$ \\
\hline 20150312 & 2457093.52 & +63 & Ekar182+AFOSC & gr4+VPH6 & $3340-9190$ & 16.44 & $2400+2400$ \\
\hline 20150328 & 2457109.59 & +79 & NOT+ALFOSC & gr4 & $3360-8920$ & 17.82 & 2400 \\
\hline 20150407 & 2457119.83 & +89 & FTN+FLOYDS & $2351 / \mathrm{mm}$ & 3180-9940 & 15.17 & 3600 \\
\hline 20150411 & 2457124.48 & +94 & Ekar $1.82 \mathrm{~m}+\mathrm{AFOSC}$ & gr4 & $3340-8150$ & 13.93 & 1800 \\
\hline 20150428 & 2457141.62 & +111 & NOT+ALFOSC & gr4 & $3250-9070$ & 14.74 & 1800 \\
\hline 20150525 & 2457168.64 & +138 & NOT+ALFOSC & gr4 & $3250-8990$ & 14.59 & 2400 \\
\hline 20150612 & 2457185.64 & +155 & NOT+ALFOSC & gr4 & $3490-9070$ & 16.36 & 1800 \\
\hline 20150720 & 2457224.39 & +193 & GTC+OSIRIS & $\mathrm{R} 1000 \mathrm{~B}+\mathrm{R} 1000 \mathrm{R}$ & $3610-10330$ & 7.98 & $900+900$ \\
\hline 20150720 & 2457224.43 & +193 & Ekar $1.82 \mathrm{~m}+\mathrm{AFOSC}$ & gr4 & $3380-8200$ & 16.34 & 1800 \\
\hline 20150806 & 2457241.32 & +210 & Ekar $1.82 \mathrm{~m}+\mathrm{AFOSC}$ & gr4 & $3380-10120$ & 14.52 & 2400 \\
\hline 20150824 & 2457259.39 & +228 & NOT+ALFOSC & gr4 & $3490-8990$ & 14.00 & 1800 \\
\hline 20150907 & 2457273.30 & +242 & Ekar $1.82 \mathrm{~m}+\mathrm{AFOSC}$ & gr4 & $3380-8200$ & 14.06 & 1800 \\
\hline 20151203 & 2457359.68 & +329 & Ekar $1.82 \mathrm{~m}+\mathrm{AFOSC}$ & gr4 & $3380-8200$ & 13.25 & 1800 \\
\hline 20160118 & 2457406.02 & +375 & FTN+FLOYDS & $2351 / \mathrm{mm}$ & $3760-9940$ & 14.89 & 3600 \\
\hline 20160318 & 2457465.60 & +435 & Ekar $1.82 \mathrm{~m}+\mathrm{AFOSC}$ & VPH6 & $3305-9240$ & 16.21 & 2700 \\
\hline 20160527 & 2457535.90 & +505 & P200+DBSP & $316 / 7500$ & $3180-10170$ & $10.30^{(\star)}$ & 840 \\
\hline 20160622 & 2457562.44 & +531 & Ekar 1.82 m+AFOSC & VPH6+VPH7 & $3600-9240$ & 15.61 & $2400+2400$ \\
\hline 20160803 & 2457603.68 & +573 & SHANE+KAST & $600 / 431+300 / 7500$ & $3420-7990$ & 6.78 & $2 \times 2700+2 \times 2700$ \\
\hline 20160820 & 2457621.38 & +590 & GTC+OSIRIS & R1000R & $5060-10330$ & 8.41 & $2 \times 1800$ \\
\hline 20160826 & 2457626.64 & +596 & P200+DBSP & $600 / 4000$ & $3300-10170$ & $10.30^{(\star)}$ & 600 \\
\hline 20160905 & 2457636.66 & +606 & SHANE+KAST & $600 / 431+300 / 7500$ & $3410-8150$ & 7.25 & 1640 \\
\hline 20160906 & 2457637.50 & +607 & Gemini N+GNIRS & HK & $8000-25000$ & $12.57^{(\star \star)}$ & $12 \times 30$ \\
\hline 20160912 & 2457644.36 & +613 & NOT+ALFOSC & gr4 & $3380-9160$ & 18.04 & 1800 \\
\hline 20161206 & 2457728.75 & +698 & $\mathrm{NOT}+\mathrm{ALFOSC}$ & gr4 & $3580-9650$ & 14.15 & 2700 \\
\hline 20161214 & 2457736.74 & +706 & NOT+ALFOSC & gr4 & $3380-9650$ & 14.08 & 2400 \\
\hline 20170127 & 2457780.98 & +750 & SHANE+KAST & $600 / 431$ & $4000-6850$ & 11.53 & 2700 \\
\hline 20170222 & 2457806.73 & +776 & NOT+ALFOSC & gr4 & $3380-9590$ & 18.08 & 2400 \\
\hline 20170315 & 2457827.57 & +797 & $\mathrm{NOT}+\mathrm{ALFOSC}$ & gr4 & $3550-9640$ & 14.58 & $2 \times 2300$ \\
\hline 20170328 & 2457841.40 & +810 & $\mathrm{NOT}+\mathrm{ALFOSC}$ & gr4 & $3380-9640$ & 14.23 & 2700 \\
\hline 20170420 & 2457863.55 & +833 & NOT+ALFOSC & gr4 & $3630-9650$ & 14.29 & $2 \times 2300$ \\
\hline 20170424 & 2457867.59 & +837 & TNG+DOLoRes & LRB+LRR & $3480-9280$ & 17.28 & $2400+2400$ \\
\hline 20170502 & 2457875.54 & +845 & TNG+DOLoRes & LRB & $3300-7990$ & 11.83 & 2400 \\
\hline 20170526 & 2457899.58 & +869 & TNG+DOLoRes & LRB+LRR & $3300-10400$ & 15.78 & $2400+2400$ \\
\hline 20170610 & 2457914.52 & +884 & TNG+DOLoRes & LRB+LRR & $3480-9240$ & 11.58 & $2400+2400$ \\
\hline 20170625 & 2457929.81 & +899 & SHANE+KAST & $600 / 431+300 / 7500$ & $3400-9930$ & 7.53 & 1200 \\
\hline 20170716 & 2457951.44 & +920 & TNG+DOLoRes & LRR & $3400-10410$ & 19.14 & 2700 \\
\hline 20170727 & 2457961.69 & +931 & SHANE+KAST & $600 / 431+300 / 7500$ & $3460-9230$ & 7.08 & 1200 \\
\hline 20170802 & 2457968.40 & +937 & TNG+DOLoRes & LRB & $3580-10400$ & 10.76 & 2700 \\
\hline 20170811 & 2457977.41 & +946 & GTC+OSIRIS & R1000B+R1000R & $3610-10330$ & 8.81 & $2 \times 1800+2 \times 1800$ \\
\hline 20180102 & 2458121.74 & +1091 & GTC+OSIRIS & R500R & $5170-9140$ & 14.64 & $2 \times 1800$ \\
\hline 20190105 & 2458489.12 & +1458 & KECK+LRIS & $400 / 3400$ & $3000-10200$ & 7.44 & 900 \\
\hline
\end{tabular}

Notes. NOT: $2.56 \mathrm{~m}$ Nordic Optical Telescope with ALFOSC; GTC: $10.4 \mathrm{~m}$ Gran Telescopio Canarias with OSIRIS; TNG: $3.56 \mathrm{~m}$ Telescopio Nazionale Galileo with DOLoRes (all located at Roque de Los Muchachos, La Palma, Spain); Ekar 1.82 m: Ekar 1.82 m Copernico Telescope with AFOSC (Mt. Ekar, Asiago, Italy); GMG24: Lijiang $2.4 \mathrm{~m}$ telescope with YFOSC (Lijiang Gaomeigu Station of Yunnan Observatories, Yunnan, China); BAO: ZTA $2.6 \mathrm{~m}$ telescope with XLT (Byurakan Astrophysical Observatory, Mt. Aragats, Armenia); SHANE: 3 m Donald Shane Telescope with KAST (Lick Observatory, San Jose, California, USA); P200: 200 inch Hale telescope with DBSP (Mt. Palomar Observatory, San Diego, California, USA); KECK: m Keck II telescope with DEIMOS and LRIS (Mauna Kea Observatory, Hawaii, USA); FTN: 2 m Faulkes Telescope North with FLOYDS, Las Cumbres Observatory node at the Haleakala Observatory, Hawaii; Gemini N: $8.1 \mathrm{~m}$ Gemini North telescope with GNIRS (Mauna Kea Observatory, Hawaii, USA). ${ }^{(\star)}$ Nominal resolution at $6562.8 \AA .{ }^{(\star \star)}$ Resolution computed around Pa $\beta$. 